\title{
Holography and Wormholes in 2+1 Dimensions
}

\author{
Kostas Skenderis $^{1,2}$, Balt C. van Rees ${ }^{1,3}$ \\ 1 Institute for Theoretical Physics, P. O. Box 94485, 1090 GL Amsterdam, The Netherlands. \\ E-mail: B.C.vanRees@uva.nl \\ 2 Korteweg-de Vries Institute for Mathematics, P. O. Box 94248, 1090 GE Amsterdam, \\ The Netherlands. E-mail: K.Skenderis@uva.nl \\ 3 YITP, State University of New York, Stony Brook, NY 11794-3840, USA
}

Received: 22 December 2009 / Accepted: 21 June 2010

Published online: 1 December 2010 - (C) The Author(s) 2010. This article is published with open access at Springerlink.com

\begin{abstract}
We provide a holographic interpretation of a class of three-dimensional wormhole spacetimes. These spacetimes have multiple asymptotic regions which are separated from each other by horizons. Each such region is isometric to the BTZ black hole and there is non-trivial spacetime topology hidden behind the horizons. We show that application of the real-time gauge/gravity duality results in a complete holographic description of these spacetimes with the dual state capturing the non-trivial topology behind the horizons. We also show that these spacetimes are in correspondence with trivalent graphs and provide an explicit metric description with all physical parameters appearing in the metric.
\end{abstract}

\section{Contents}

1. Introduction and Summary of Results . . . . . . . . . . . . . . . . . . . 584

2. Lorentzian Wormholes . . . . . . . . . . . . . . . . . . . 588

2.1 Wormholes as quotient spacetimes . . . . . . . . . . . . . . . . 588

2.2 Physical properties . . . . . . . . . . . . . . . . . . . 591

3. Euclidean Wormholes . . . . . . . . . . . . . . . . . . . 592

3.1 Construction . . . . . . . . . . . . . . . . . 592

4. Holographic Interpretation of Euclidean Wormholes . . . . . . . . . . . . . 594

4.1 Bulk interpretation and relation to Teichmüller theory . . . . . . . . . . 597

4.2 Non-handlebodies . . . . . . . . . . . . . . . . . . . . . . . 597

5. Holographic Interpretation of Lorentzian Wormholes . . . . . . . . . . . . . 599

5.1 Naive computation . . . . . . . . . . . . . . . . . 599

5.2 Lorentzian gauge/gravity prescription . . . . . . . . . . . . . . . . . . 599

5.3 Gauge/gravity duality for Lorentzian wormholes . . . . . . . . . . . . . 601

5.4 More fillings . . . . . . . . . . . . . . . . . . . 605

5.5 State dual to wormholes . . . . . . . . . . . . . . . . 606

5.6 2-point functions . . . . . . . . . . . . . . . . . 607 


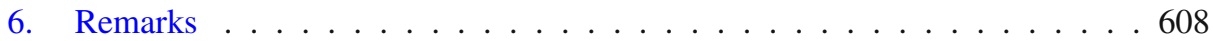

6.1 Other bulk spacetimes . . . . . . . . . . . . . . . . . . . 608 608

6.2 Rotating wormholes . . . . . . . . . . . . . . . . . . . . 609

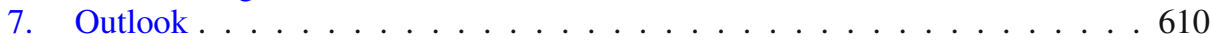

A. Coordinate Systems . . . . . . . . . . . . . . . . 6 611

A.1 Fenchel-Nielsen coordinates . . . . . . . . . . . . . 611

A.2 Construction of the charts . . . . . . . . . . . . . . . 613

A.3 Parameters . . . . . . . . . . . . . . . . . . . . 619

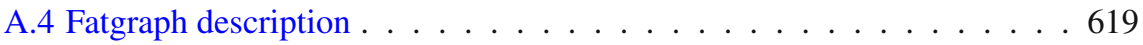

A.5 Transition functions . . . . . . . . . . . . . . . . . . . 620

B. Eternal Black Holes and Filled Tori . . . . . . . . . . . . . . . . . 623

\section{Introduction and Summary of Results}

The gauge/gravity duality [1-3] has significantly enhanced our understanding of gravity and gauge theory. This can be ascribed largely to a well developed dictionary that translates results between string and gauge theory. Although the entries in the dictionary are by now well understood for Euclidean backgrounds, a real-time dictionary along the lines of [3] was developed only recently in [4,5]. This real-time dictionary uses a construction that is a holographic version of the closed time path method of non-equilibrium QFT [6-9] and results in a prescription that incorporates in the bulk the information about the QFT initial and final states via a Hartle-Hawking type construction $[10,11]$. Thus this prescription although originated from QFT considerations is also in line with expectations from quantum gravity.

In this paper we apply the prescription of $[4,5]$ to a class of 2+1-dimensional 'wormhole' spacetimes that were found and studied in [12,13]. Our main motivation is to investigate global issues in gauge/gravity duality. Three dimensional gravity is an ideal setup to study this problem because of the absence of local degrees of freedom. In the holographic context one finds that the general solution of the bulk Einstein equations with a cosmological constant in the Fefferman-Graham gauge can be explicitly obtained for general Dirichlet boundary conditions specified by an arbitrary boundary metric [14]. In contrast to the higher dimensional case, where in general the Fefferman-Graham expansion contains an infinite number of terms, in three dimensions the series terminates (see (33)) and all coefficients can be expressed explicitly in terms of the boundary metric and boundary stress energy tensor. What is left to be done is to impose regularity in the interior and this step requires global analysis. ${ }^{1}$

The wormholes are global solutions of $2+1$ dimensional gravity with a negative cosmological constant. They can be thought of as generalized eternal BTZ black holes. Whereas the spatial slices of an eternal BTZ black hole have a cylindrical topology, in the wormholes the spatial slices are general two-dimensional Riemann surfaces with boundary. We sketch an example of a wormhole in Fig. 1. These spacetimes have a number of different asymptotic regions, which we will call outer regions in this paper, one for each boundary component of the Riemann surface. The outer regions are separated by horizons and there is a non-trivial topology behind the horizons. The wormholes are locally $\mathrm{AdS}_{3}$ and should have a holographic interpretation. Each outer region, however, is isometric to the static BTZ black hole and it would seem as though holographic data,

\footnotetext{
1 Note also that the Fefferman-Graham coordinates are in general well-defined only in a neighborhood of the boundary and they may not cover the entire spacetime.
} 


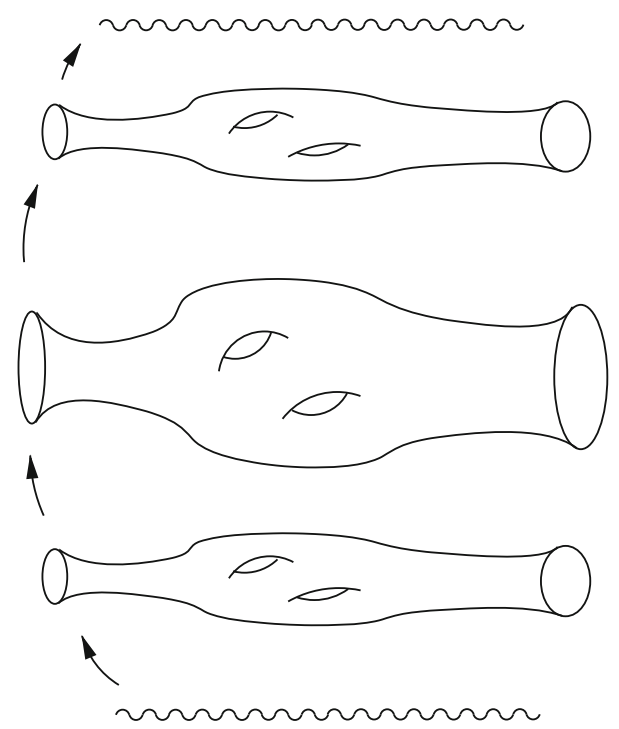

Fig. 1. A wormhole spacetime with two outer regions corresponding to a Riemann surface of genus 2 with 2 boundary components

which are obtained from the behavior of the solution near the conformal boundary, do not contain enough information to completely describe the wormhole spacetime. This follows from a simple counting argument. The spacetimes are uniquely determined given a Riemann surface of genus $g$ with $m$ boundaries. Such a Riemann surface is determined by $6 g-6+3 m$ parameters. Each of the outer regions however depends on only one parameter, the mass of the BTZ black hole, so the holographic data from the $m$ outer regions would seem to provide only $m$ parameters. We will shortly describe how the real-time dictionary resolves this puzzle.

There are corresponding Euclidean solutions which have been discussed in [15]. These spaces are handlebodies, i.e. closed surfaces of genus $g$ filled in with hyperbolic three-space. These are also generalizations of BTZ whose Euclidean counterpart is a solid torus, i.e. a handlebody of genus 1 . For these spacetimes a fairly straightforward application of the Euclidean gauge/gravity prescription shows there is no corresponding puzzle: the holographic one-point function captures the non-trivial topology and in particular does contain enough parameters to completely describe these spaces. This indicates that it is the real-time issues that are crucial in understanding holography for the Lorentzian wormholes.

We will indeed find that once we properly apply the real-time gauge/gravity prescription of $[4,5]$ there is a direct and unambiguous holographic interpretation of the entire Lorentzian wormhole spacetimes. The real-time prescription relies on gluing to a given Lorentzian spacetime Euclidean spaces that provide the initial and final states. A class of such Euclidean spaces are the handlebodies described above, but we emphasize that there are also other choices one can make. Once the complete spacetime has been specified (with the Euclidean parts representing initial/final states included), the holographic one-point functions do carry enough information about the spacetime and in particular the geometry behind the horizons. This information is encoded in the initial and final states. 
The way this happens is instructive and reflects a number of subtle points about the holographic dictionary. Recall that because of the holographic conformal anomaly $[16,17]$ the theory depends on the specific boundary metric, not just its conformal class. In particular, the expectation value of the stress energy tensor changes anomalously under bulk diffeomorphisms that induce a boundary Weyl transformation [18,19]. Now as mentioned earlier, one can choose coordinates such that the metric in any of the outer regions of the wormhole is exactly that of the BTZ black hole. In these coordinates the boundary metric is flat. According to the prescription of $[4,5]$, however, the Lorentzian solution should be matched in a smooth fashion to a corresponding Euclidean solution. Euclidean solutions that satisfy all matching conditions are provided by the handlebodies but these can never have a boundary metric that is globally flat (because the Euler number of the boundary Riemann surface is negative). One can arrange for an everywhere smooth matching by performing a bulk diffeomorphism on the Lorentzian side that induces an appropriate boundary Weyl transformation such that the Lorentzian boundary metric now matches with that of the handlebody. This has the effect that the expectation value of the stress energy tensor changes from its BTZ value to a new value, which is smooth as we cross from the Euclidean side to the Lorentzian side (as it should be [5]). In other words, the initial state via the matching conditions dictates a specific bulk diffeomorphism on the outer regions of the Lorentzian solution and as a result the holographic data extracted using the solution in this coordinate system encode the information hidden behind the horizon.

Our results indicate that the dual state for a wormhole with $n$ outer regions is an entangled state in a Hilbert space that is the direct product of $n$ Hilbert spaces, one for each component. A reasonable guess for this state is that it is the state obtained by the Euclidean path integral over the conformal boundary of half of the Euclidean space glued at the $t=0$ surface of the Lorentzian wormhole. This is a Riemann surface with $n$ boundaries and in the case of the handlebodies discussed above, it is precisely the Riemann surface that serves as the $t=0$ slice of the wormhole. If one traces out all components but one, then the reduced description is given in terms of a mixed state in the remaining copy.

This paper is organized as follows. In the next section we describe the wormhole spacetimes in detail and in Sect. 3 and we discuss the handlebodies. In Sect. 4 and 5 we discuss holography for the handlebodies and the Lorentzian wormholes, respectively. We emphasize that our analysis applies only to non-rotating wormholes. The interesting possibility of extending the analysis to rotating wormholes is discussed in Sect. 6 along with several other general remarks. We conclude in Sect. 7 with an outlook.

In all of the previous literature and in the main text of this paper the wormhole spacetimes are described abstractly as quotients of a domain in $\mathrm{AdS}_{3}$. While this presents no loss of information, this description is abstract and requires mastering prerequisite mathematical background in order to understand the properties of these spacetimes. One should contrast this with the case of the BTZ black hole [20,21] where one has an explicit metric containing the physical parameters (the mass and angular momentum). The BTZ also has an abstract representation as a quotient of a domain of $\mathrm{AdS}_{3}$ but this has not been used as much as the explicit metric description. With the hope that a more concrete description of the wormholes would make them more readily accessible we derive in Appendix A an explicit metric description where all parameters that determine the spacetime appear in the metric and we summarize this result here.

All information about the wormhole can be summarized in an oriented trivalent fatgraph, like the one in Fig. 2. For a wormhole that is based on a Riemann surface of genus 


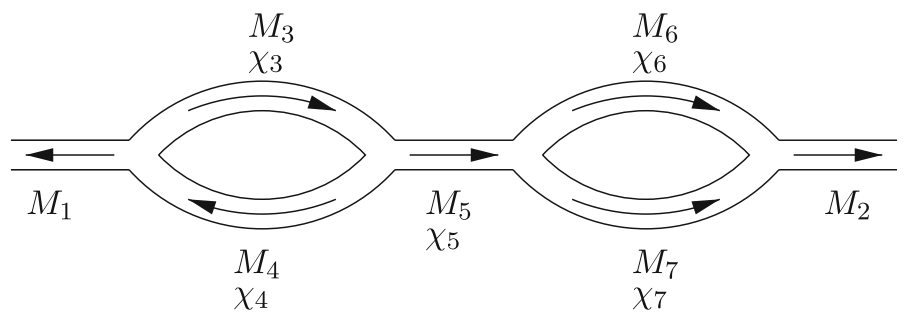

Fig. 2. A fatgraph representing the wormhole spacetime sketched in Fig. 1

$g$ with $m$ boundaries, this graph should have $m$ outer edges (ends) and $(3 g-3+m)$ inner edges. With every outer edge we associate one parameter $M_{k}$ and with every inner edge two parameters $M_{i}, \chi_{i}$, where $k=1, \ldots, m$ and $i=m+1, \ldots, 3 g-3+2 m$. This yields a total of $(6 g-6+3 m)$ parameters, which is indeed the correct number of moduli for a Riemann surface of genus $g$ with $m$ boundaries. ${ }^{2}$ We now associate a coordinate chart for every edge of the fatgraph and every such chart has a canonical metric on it. The precise definition of the coordinate charts as well as the meaning of the orientation is given in Appendix A. To complete the description we need to specify the transition functions in the overlap regions and these are also given in Appendix A.

Thus, the spacetime is described by the graph and two different metrics, one for the outer charts and one for the inner charts. The metric in the $k^{\text {th }}$ outer chart takes the form:

$$
d s_{k}^{2}=\frac{\rho^{2}+M_{k}}{\cosh ^{2}\left(\sqrt{M_{k}} \tilde{\tau}\right)}\left(-d \tilde{\tau}^{2}+d \varphi^{2}\right)+\frac{d \rho^{2}}{\rho^{2}+M_{k}} .
$$

The corresponding $(\tilde{\tau}, \rho, \varphi)$ coordinate system has coordinate ranges,

$$
\tilde{\tau} \in \mathbb{R}, \quad \varphi \sim \varphi+2 \pi, \quad \frac{\cosh \left(\sqrt{M_{k}} \tilde{\tau}\right) \rho}{\sqrt{\rho^{2}+M_{k}}}>-\frac{\beta^{2}}{1+\beta^{2}},
$$

where $\beta$ is defined in Appendix A. These coordinates extend beyond the future and past horizons, which lie at

$$
\rho=\sqrt{M_{k}}\left|\sinh \left(\sqrt{M_{k}} \tilde{\tau}\right)\right|
$$

If we restrict ourselves to the region outside of the horizons we may also put the metric in the static BTZ form,

$$
d s_{k}^{2}=-\left(r^{2}-M_{k}\right) d t^{2}+\frac{d r^{2}}{r^{2}-M_{k}}+r^{2} d \phi^{2},
$$

with coordinate ranges, $r>M_{k}, t \in \mathbb{R}$ and $\phi \sim \phi+2 \pi$. In these metrics $M_{k}$ is the parameter of the corresponding outer edge. The metric in the $i^{\text {th }}$ inner chart is given by

$$
\begin{gathered}
d s_{i}^{2}=\frac{1}{\cosh ^{2}(t)}\left(-d t^{2}+\frac{\mu_{i}^{2} d r^{2}}{\left(\mu_{i} r+v_{i}\right)^{2}+\cos ^{2}\left(\chi_{i}\right)}+M_{i}\left(1+\left(\mu_{i} r+v_{i}\right)^{2}\right) d \psi^{2}\right. \\
\left.-\frac{2 \mu_{i} \sqrt{M}_{i} \sin \left(\chi_{i}\right)}{\sqrt{\left(\mu_{i} r+v_{i}\right)^{2}+\cos ^{2}\left(\chi_{i}\right)}} d \psi d r\right)
\end{gathered}
$$

2 As we review in Appendix A, the parameters $\left\{M_{I}, \chi_{i}\right\}(I=k, i)$ are directly related to the Fenchel-Nielsen coordinates of the moduli space of the Riemann surface. 
with coordinate ranges, $r \in[-1,1], \tau \in \mathbb{R}$ and $\psi \sim \psi+2 \pi$. This is a time-dependent metric of constant negative curvature which (as far as we know) has not appeared before in the literature. The parameters $M_{i}$ and $\chi_{i}$ are the parameters associated with the $i^{\text {th }}$ inner edge. The parameters $\mu_{i}, v_{i}$ on the other hand are functions of the $M$ parameters, see the discussion in Sect. A.3. Note that both metrics (1) and (5) have a $U$ (1) isometry, the transition functions however do not respect this symmetry and the entire spacetime is not $U(1)$ symmetric.

\section{Lorentzian Wormholes}

In this section we describe the Lorentzian wormholes. We show how they can be obtained as quotients of a part of $\mathrm{AdS}_{3}$ and discuss their physical properties. The material in this section summarizes discussions in [12,13,22-24]. We will occasionally use results from Teichmüller theory; more information on this topic can be found in [24-27].

2.1. Wormholes as quotient spacetimes. The wormholes are obtained as follows. One starts with a Riemann surface $S$ which is a quotient of the upper half plane $H$ with respect to some discrete subgroup $\Gamma$ of $S L(2, \mathbb{R})$. The upper half plane is then embedded into $\mathrm{AdS}_{3}$ and the action of $\Gamma$ is extended to $\mathrm{AdS}_{3}$ entirely. After removing certain regions in $\mathrm{AdS}_{3}$ that would lead to pathologies, one may take the quotient of the remainder with respect to $\Gamma$, which will give us the wormhole spacetime we are after. The topology of such a spacetime is $S \times \mathbb{R}$, with $S$ the Riemann surface we started with and $\mathbb{R}$ the time direction. The aim of this subsection is to discuss this procedure in more detail.

2.1.1. Riemann surfaces. Consider a Riemann surface $S$ with $m>0$ circular boundaries but no punctures. ${ }^{3}$ As follows from the uniformization theorem, such a Riemann surface can be described as a quotient of the upper half plane $H$ by some discrete subgroup $\Gamma$ of $\operatorname{PSL}(2, \mathbb{R})$ :

$$
S=H / \Gamma
$$

where the action of

$$
\left(\begin{array}{ll}
a & b \\
c & d
\end{array}\right) \in P S L(2, \mathbb{R}) \equiv S L(2, \mathbb{R}) /\{ \pm \mathbf{1}\}
$$

on $H$ is given by

$$
z \mapsto \frac{a z+b}{c z+d}
$$

Since these transformations act as isometries for the standard negatively curved metric on $H$,

$$
d s^{2}=\frac{d z d \bar{z}}{\operatorname{Im}(z)^{2}}
$$

this metric descends to a metric on $S$. Up to a constant rescaling, this is the unique hermitian metric of constant negative curvature on $S$ (given the complex structure of $S$ )

\footnotetext{
3 Recall that a Riemann surface is a topological two-dimensional surface equipped with a complex structure. One can distinguish between punctures and circular boundaries precisely because of the complex structure.
} 


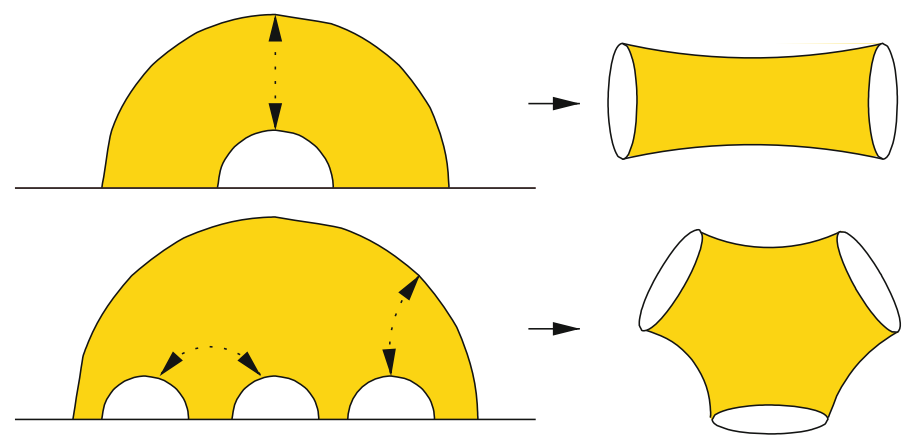

Fig. 3. On the left we sketched two fundamental domains in $H$. The boundaries are pairwise glued together as indicated by the arrows. After the gluing we find the Riemann surfaces shown on the right

and $\Gamma$ is unique up to conjugation. We shall require absence of conical singularities on $S$, which means that the nontrivial elements of $\Gamma$ cannot have fixed points in $H$. A simple analysis of the fixed points of (8) tells us that we should require that for all elements $\gamma \in \Gamma$ we have

$$
|a+d| \geq 2 \text {. }
$$

Furthermore, absence of any punctures on $S$ translates into $|a+d|>2$ for all nontrivial $\gamma$. We then say that $\Gamma$ consists of only hyperbolic elements (and the identity), and we call it a Fuchsian group of the second kind.

A particularly convenient way to visualize $S$ as a quotient of $H$ is to define a fundamental domain in $H$, basically a domain in $H$ whose boundary in $H$ consists of various segments that are pairwise identified by generators of $\Gamma$. For convenience we may take these segments to be geodesic segments, which are circular arcs in $H$. Two examples of a fundamental domain are sketched in Fig. 3.

From the theory of Fuchsian groups we obtain that the fixed points of such a group $\Gamma$ form a nowhere dense subset of the conformal boundary $\partial H$ of $H$, which is the real line plus a point at infinity. We will call this set the limit set and denote it as $\Lambda(\Gamma)$. Notice that $\Lambda(\Gamma)$ is invariant under the action of $\Gamma$.

2.1.2. $A d S_{3}$. To find the wormhole spacetime associated to $S$, we first fix some coordinate systems and conventions for $\mathrm{AdS}_{3}$. We define $\mathrm{AdS}_{3}$ as the surface

$$
-U^{2}-V^{2}+X^{2}+Y^{2}=-1,
$$

in $\mathbb{R}^{2,2}$, where the metric has the form

$$
d s^{2}=-d U^{2}-d V^{2}+d X^{2}+d Y^{2},
$$

and we have set the AdS radius $\ell^{2}=1$. By combining $(U, V, X, Y)$ into a matrix,

$$
\left(\begin{array}{cc}
V+X & Y+U \\
Y-U & V-X
\end{array}\right)
$$

we may identify the hyperboloid with the space of real unit determinant matrices, i.e. the group $S L(2, \mathbb{R})$. The connected component of the identity of the isometry group of $\mathrm{AdS}_{3}$,

$$
\operatorname{Isom}_{0}\left(\mathrm{AdS}_{3}\right)=(S L(2, \mathbb{R}) \times S L(2, \mathbb{R})) / \mathbb{Z}_{2},
$$


acts by left and right multiplication: if $\left(\gamma_{1}, \gamma_{2}\right) \in S L(2, \mathbb{R}) \times S L(2, \mathbb{R})$ then their action on $\mathrm{AdS}_{3}$ is defined by

$$
\left(\begin{array}{cc}
V+X & Y+U \\
Y-U & V-X
\end{array}\right) \mapsto \gamma_{1}\left(\begin{array}{cc}
V+X & Y+U \\
Y-U & V-X
\end{array}\right) \gamma_{2}^{T} .
$$

Taking the transpose of $\gamma_{2}$ is a convention which will turn out to be convenient below.

We may describe a patch in the hyperboloid with Poincaré coordinates $(t, x, y)$ defined by

$$
t=\frac{U}{V-X}, \quad x=\frac{Y}{V-X}, \quad y=\frac{1}{V-X} .
$$

In these coordinates, the metric takes the form

$$
d s^{2}=\frac{-d t^{2}+d x^{2}+d y^{2}}{y^{2}} .
$$

Although the Poincaré coordinate system may not cover the entire region of interest, the coordinate horizon at $y \rightarrow \infty$ will not be important in what follows.

2.1.3. Constructing wormholes. We can now construct a three-dimensional wormhole spacetime from the Riemann surface $S=H / \Gamma$. We begin by extending the action of the isometries of $H$ to isometries on $\mathrm{AdS}_{3}$ via the homomorphism:

$$
\operatorname{PSL}(2, \mathbb{R}) \hookrightarrow(S L(2, \mathbb{R}) \times S L(2, \mathbb{R})) / \mathbb{Z}_{2},
$$

which is given explicitly by $\operatorname{PSL}(2, \mathbb{R}) \ni \gamma \mapsto(\gamma, \gamma) \in S L(2, \mathbb{R}) \times S L(2, \mathbb{R})$. One may check that elements of the form $(\gamma, \gamma)$ leave the slice $U=0$ invariant when they act on $\mathrm{AdS}_{3}$ according to (15). Furthermore, their action on the slice $U=0$ is exactly of the form (8) when we define $z=x+i y$ with $(x, y)$ the Poincaré coordinates on this slice.

The image of $\Gamma$ under this homomorphism is a discrete subgroup of $\operatorname{Isom}_{0}\left(\mathrm{AdS}_{3}\right)$ which is isomorphic to $\Gamma$ and which we denote as $\hat{\Gamma}$. One may now try to take a quotient like $\mathrm{AdS}_{3} / \hat{\Gamma}$, which clearly contains $S=H / \Gamma$ as the slice given by $U=0$. However, away from the slice $U=0$ this quotient turns out to have closed null or timelike curves. To get a spacetime free of pathologies we proceed as follows.

The embedding of $H$ in $\mathrm{AdS}_{3}$ as the slice $U=0$ can be directly extended to an embedding of $\partial H$ in the conformal boundary of $\mathrm{AdS}_{3}$. This extension maps the limit set $\Lambda(\Gamma)$ to a subset of the conformal boundary of $\mathrm{AdS}_{3}$, which we denote as $\Lambda(\hat{\Gamma})$. We then pass to the universal covering space of the hyperboloid and remove from it all points with a timelike or lightlike separation to $\Lambda(\hat{\Gamma})$ (after a standard conformal rescaling of the metric that brings the radial boundary to finite distance). Informally speaking, we are removing the filled forward and backward semi-lightcones emanating from every point in $\Lambda(\hat{\Gamma})$. We call the remainder $\widehat{\mathrm{AdS}}_{3}$ which notably includes the original slice $U=0$ entirely. The elements of $\hat{\Gamma}$ leave $\Lambda(\hat{\Gamma})$ invariant and, being isometries, they map lightcones to lightcones so they also leave $\widehat{\mathrm{AdS}}_{3}$ invariant. Furthermore, the quotient

$$
M=\widehat{\mathrm{AdS}}_{3} / \hat{\Gamma}
$$

is a spacetime that is free of closed timelike curves and conical singularities $[22,23]$ and contains $S=H / \Gamma$ as a hypersurface. These spacetimes are what we call the $2+1-$ dimensional wormholes. 
2.2. Physical properties. We briefly discuss some physical properties of the wormholes. First of all, they are of course locally $\mathrm{AdS}_{3}$ but, as was mentioned above, their global topology is of the form $S \times \mathbb{R}$ with $S$ a surface with $m>0$ circular boundaries and $\mathbb{R}$ representing time. We sketched an example in Fig. 1, where $S$ has genus 2 and has 2 boundary components. The wormholes can have an arbitrary number $m>0$ cylindrical boundaries, and $S$ can have arbitrary genus $g \geq 0$. There are two special cases: when $m=2$ and $g=0$ we obtain the eternal static BTZ black hole and the case $m=1, g=0$ is just AdS.

Except for the eternal BTZ black hole described already in [21], none of the wormholes have globally defined Killing vector fields since no such isometry of $\mathrm{AdS}_{3}$ commutes with all the elements in $\Gamma$. On the other hand, all wormholes admit a discrete $\mathbb{Z}_{2}$ isometry, which acts as time reflection $U \leftrightarrow-U$ and therefore leaves the $U=0$ slice invariant. The wormholes are not geodesically complete and begin with and end on locally Milne-type singularities. Furthermore, these singularities have associated black and white hole horizons (not drawn in Fig. 1).

Perhaps surprisingly, the $m$ segments of the spacetime between the horizons and the conformal boundaries are exactly the same as for the BTZ black hole [12]. More precisely, we find that these segments can be covered by a $(t, r, \phi)$ coordinate system with the coordinate ranges $r>M, t \in \mathbb{R}$ and $\phi \sim \phi+2 \pi$, in which the metric is of the form

$$
d s^{2}=-\left(r^{2}-M\right) d t^{2}+\frac{d r^{2}}{r^{2}-M}+r^{2} d \phi^{2} .
$$

The mass $M$ can be different for the $m$ different boundaries, but it should always be strictly positive so we do not 'pinch off' the rest of the wormhole. We will call these $m$ segments the outer regions of the wormhole, and what remains when we excise these segments we call the inner region. Notice that what we call the outer region is precisely the domain of outer communication [12]. What was called the 'exterior region' in [12] is obtained by keeping only the region outside of the future horizon, but we will never consider this region here. The fact that the nontrivial topology is hidden behind the horizons is in agreement with the general discussion of [28].

Depending on the genus of $S$, the geometry in the inner region is specified by a discrete number of parameters, namely the moduli of $S$. One may for example think of these parameters as the elements $\left(a_{i}, b_{i}, c_{i}, d_{i}\right)$ of a set $\left\{\gamma_{i}\right\}$ of generators of $\Gamma$. It will be important for what follows to notice that these parameters do not show up in the metric on the outer regions if we put the metric in the form (20). On the other hand, in Appendix A we present a set of different coordinate systems that can be used to describe the wormholes as well. In these coordinate systems the coordinate ranges are natural and the metric features several parameters that are geometric (rather than abstract matrix elements). For example, some of the parameters are directly related to the lengths of certain cycles on the surface. As we explain in more detail in Appendix A, the combination of all parameters from the different charts that make up the surface can be used to completely describe the spacetime. ${ }^{4}$

It is straightforward to embed the wormholes into string theory, since the wormholes are locally just $\mathrm{AdS}_{3}$. For example, a wormhole times $S^{3} \times T^{4}$ with a constant dilaton and three-form flux is an asymptotically locally $\mathrm{AdS}_{3}$ solution of type IIB supergravity. However, these solutions are not supersymmetric.

\footnotetext{
4 The parameters $\left(a_{i}, b_{i}, c_{i}, d_{i}\right)$ are similar to the Fricke coordinates on the moduli space of $S$, whereas the metric we find in Appendix A features parameters that are similar to Fenchel-Nielsen coordinates on the moduli space of $S$. These coordinate systems on the moduli or rather Teichmüller space of $S$ are described in more detail in for example [25].
} 


\section{Euclidean Wormholes}

In this section we describe 'Euclidean wormholes'. These Euclidean spaces are handlebodies and one may think of them as closed Riemann surfaces filled in with three-dimensional hyperbolic space. They are a natural generalization of the Euclidean BTZ black hole, which is a solid torus [29]. These spaces were considered first in a holographic context in [15], where it was argued that they are natural Euclidean analogues of the Lorentzian wormholes, even though they are not obtained by analytic continuation of a globally defined time coordinate. We will see later that they are indeed suitable Euclidean counterparts of the Lorentzian wormholes, in the sense of the real-time gauge/gravity prescription of [4,5], but we will also show that they are not the only possible Euclidean counterparts.

3.1. Construction. We will again describe the handlebodies via a quotient construction. Recall that Euclidean (unit radius) $\mathrm{AdS}_{3}$, denoted by $H^{3}$, is defined as the hyperboloid

$$
U^{2}-V^{2}+X^{2}+Y^{2}=-1,
$$

with $V>0$ in $\mathbb{R}^{1,3}$ with the metric:

$$
d s^{2}=d U^{2}-d V^{2}+d X^{2}+d Y^{2} .
$$

We may again combine $(U, V, X, Y)$ into a matrix:

$$
\left(\begin{array}{cc}
V+X & Y+i U \\
Y-i U & V-X
\end{array}\right)
$$

which maps $H^{3}$ into the space of hermitian unit determinant matrices. An element $\gamma$ of the connected component of the identity of the isometry group of $H^{3}$,

$$
\operatorname{Isom}_{0}\left(H^{3}\right)=\operatorname{PSL}(2, \mathbb{C}),
$$

acts on $H^{3}$ as

$$
\left(\begin{array}{cc}
V+X & Y+i U \\
Y-i U & V-X
\end{array}\right) \mapsto \gamma\left(\begin{array}{cc}
V+X & Y+i U \\
Y-i U & V-X
\end{array}\right) \gamma^{\dagger}
$$

Notice that $P S L(2, \mathbb{C})$ maps the upper hyperboloid to itself.

We may again define Poincaré coordinates $(\tau, x, y)$ via

$$
\tau=\frac{U}{V-X}, \quad x=\frac{Y}{V-X}, \quad y=\frac{1}{V-X} .
$$

In these coordinates, the metric takes the form

$$
d s^{2}=\frac{d \tau^{2}+d x^{2}+d y^{2}}{y^{2}} .
$$

This time there are no coordinate singularities and this metric covers all of $H^{3}$.

To find the Euclidean analogue of the wormholes, we again start with the Riemann surface $S=H / \Gamma$. The action of $\Gamma$ on $H$ can again be extended to an action on $H^{3}$ entirely, this time via the trivial homomorphism

$$
\operatorname{PSL}(2, \mathbb{R}) \hookrightarrow \operatorname{PSL}(2, \mathbb{C}),
$$



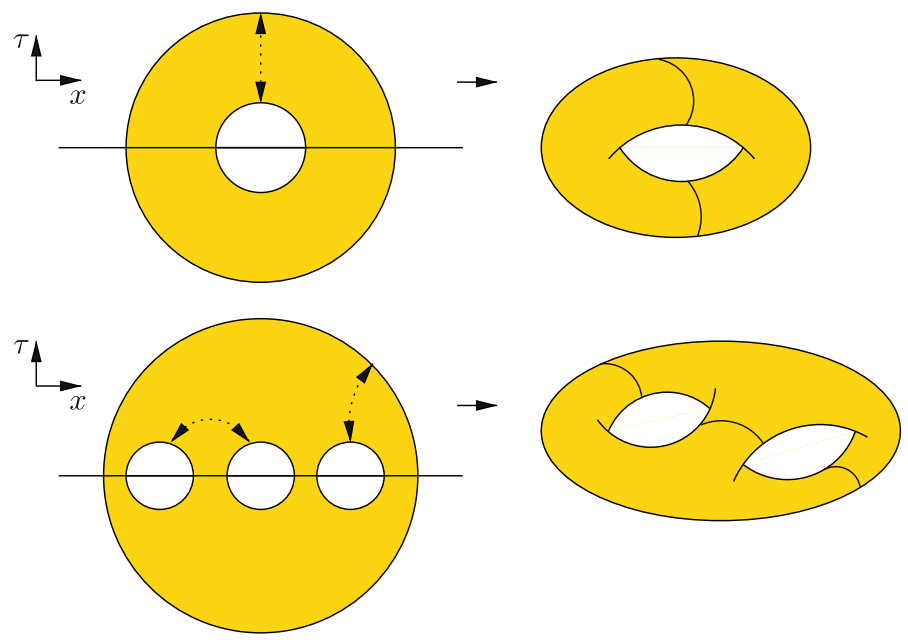

Fig. 4. The Schottky double of the Riemann surfaces of Fig. 3 is constructed by gluing two copies of the fundamental domain to each other and identifying the boundaries. The line $\tau=0$ is invariant and the Schottky double surface is symmetric under reflection in this line. The limit set $\Lambda(\hat{\Gamma})$ is a subset of the line $\tau=0$ but is not shown here. It has to be removed from the $(\tau, x)$ plane before taking a quotient

(i.e. any element of $\operatorname{PSL}(2, \mathbb{R})$ is also an element of $P S L(2, \mathbb{C}))$. One may again check that real elements in $\operatorname{PSL}(2, \mathbb{C})$ leave the slice $U=0$ invariant when they act on $H^{3}$ according to (25). Furthermore, their action on the slice $U=0$ is again of the form (8) if we define $z=x+i y$ with $(x, y)$ the Poincaré coordinates on this slice.

After using this homomorphism to map $\Gamma$ to $\hat{\Gamma}$ in $\operatorname{Isom}_{0}\left(H^{3}\right)$, we can define the quotient

$$
M_{e}=H^{3} / \hat{\Gamma},
$$

which now never leads to pathologies; $M_{e}$ is a smooth and geodesically complete manifold. This quotient again contains $S=H / \Gamma$ as the $U=0$ slice, and $M_{e}$ also admits a $\mathbb{Z}_{2}$ isometry that leaves this surface invariant.

Let us now show why we call $M_{e}$ a handlebody. We can extend the action of $\hat{\Gamma}$ to the conformal boundary of $H^{3}$ which is an $S^{2}$. Consider an element $\gamma$ of $\hat{\Gamma}$, i.e. a real element of $P S L(2, \mathbb{C})$, acting as (8) on the $U=0$ slice. Its extension to $H^{3}$ entirely is found most easily by noticing that, according to (25), real elements of $\operatorname{Isom}_{0}\left(H^{3}\right)$ leave slices of constant $U=\tau / y$ invariant and act on these slices exactly as on the slice $U=0$. In the limit where $y \rightarrow 0$, we recover the action of $\gamma$ on the conformal boundary, which is just the same as on the slice $U=0$,

$$
\gamma: w \mapsto \frac{a w+b}{c w+d}
$$

but this time with $w=x+i \tau$.

From (30) we find that the great circle $\tau=0$ is invariant because $a, b, c, d$ in (30) are all real. Just as in the Lorentzian case, this circle contains the limit set $\Lambda(\hat{\Gamma})$. After removing the limit set, the quotient of the remainder $S^{2} \backslash \Lambda(\hat{\Gamma})$ with respect to $\hat{\Gamma}$ is a smooth manifold. As can be seen from Fig. 4, it consists of two copies of $S$, one from 


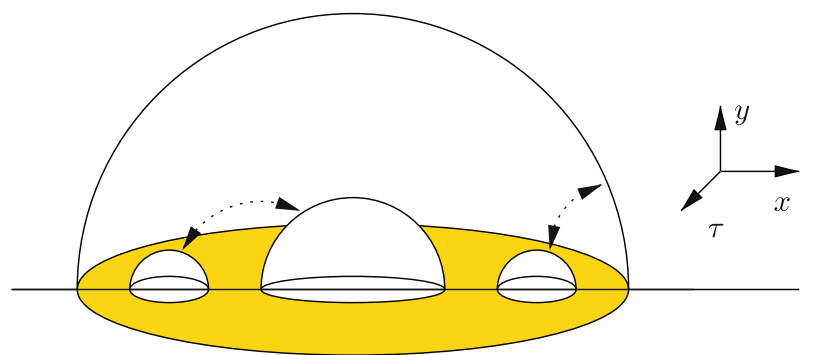

Fig. 5. The extension of the fundamental domain for $\hat{\Gamma}$ from the $S^{2}$ to $H^{3}$ is bounded by a set of hemispheres that should be pairwise identified. We recover $S$ as the surface given by $\tau=0$

the upper and one from the lower half plane, glued together along their $m$ boundaries. This surface is called the Schottky double $S_{d}$ of $S$. If $S$ has genus $g$ and $m$ holes, then $S_{d}$ has genus $2 g+m-1$ and no holes. Since $S_{d}$ is just the conformal boundary of $M_{e}$, we may think of $M_{e}$ as a filled $S_{d}$. This shows that $M_{e}$ is indeed a handlebody.

A fundamental domain for $M_{e}$ in $H^{3}$ is sketched in Fig. 5 and can be found by extending the circles on the boundary $S^{2}$ to hemispheres in $H^{3}$. The fundamental domain for the original surface $S$ is then embedded in this three-dimensional fundamental domain as the surface given by $\tau=0$.

\section{Holographic Interpretation of Euclidean Wormholes}

We discuss in this section the holographic interpretation of the Euclidean wormholes. Our discussion, which builds on [15,24,30,31], is a fairly straightforward application of Euclidean holography. In the next section we will turn to Lorentzian wormholes, where things are more subtle.

Recall that the boundary $S_{d}$ of the handlebody is a closed Riemann surface with $g>1$ and therefore naturally has a metric of constant negative curvature. Below, following $[24,30]$, we holographically compute the one-point function of the stress energy tensor for this background metric.

The negative curvature metric on $S_{d}$ is obtained by describing $S_{d}$ as a quotient of $H$, that is $S_{d}=H / \Gamma_{d}$. Above we described $S_{d}$ as a quotient of the conformal boundary $S^{2}$ of $H^{3}$, with the limit set $\Lambda(\hat{\Gamma})$ removed, with respect to the group $\hat{\Gamma}$, that is $S_{d}=\left(S^{2} \backslash \Lambda(\hat{\Gamma})\right) / \hat{\Gamma}$. As sketched in Fig. 6, this is just a different description of the same Riemann surface. Therefore, there should be a locally biholomorphic map $J: H \rightarrow S^{2}$ between the two descriptions. Such a map should be compatible with the actions of $\Gamma_{d}$ and $\Gamma$, in the sense that for every $\gamma_{d} \in \Gamma_{d}$ there should exist a $\gamma \in \hat{\Gamma}$ such that $J \circ \gamma_{d}=\gamma \circ J$. Now consider the case where $\gamma$ is trivial for a nontrivial $\gamma_{d}$. Since $\gamma_{d}$ corresponds to a nontrivial one-cycle on $S_{d}$, the image of this one-cycle under $J$ must be a nontrivial closed curve on $S^{2} \backslash \Lambda(\hat{\Gamma})$. The only way to do this is to let this curve encircle a nonempty subset of $\Lambda(\hat{\Gamma})$ on the $S^{2}$, but such a one-cycle is contractible in the bulk manifold. For example, the dashed circle drawn within the fundamental domain of Fig. 6 can be continuously shrunk to a point by moving it inside the bulk, as can be seen from Fig. 5. Therefore, precisely those $\gamma_{d}$ for which $J \circ \gamma_{d}=J$ correspond to contractible cycles in the bulk. The map $J$ thus determines the filling of $S_{d}$ : different maps $J$ (up to composition with an element of $\operatorname{PSL}(2, \mathbb{R})$ or $\operatorname{PSL}(2, \mathbb{C})$ ) precisely correspond to the different fillings of $S_{d}$. It therefore suffices to know $J$ in order to know which cycles of $S_{d}$ are filled to give a handlebody and therefore to determine the Euclidean bulk geometry. 


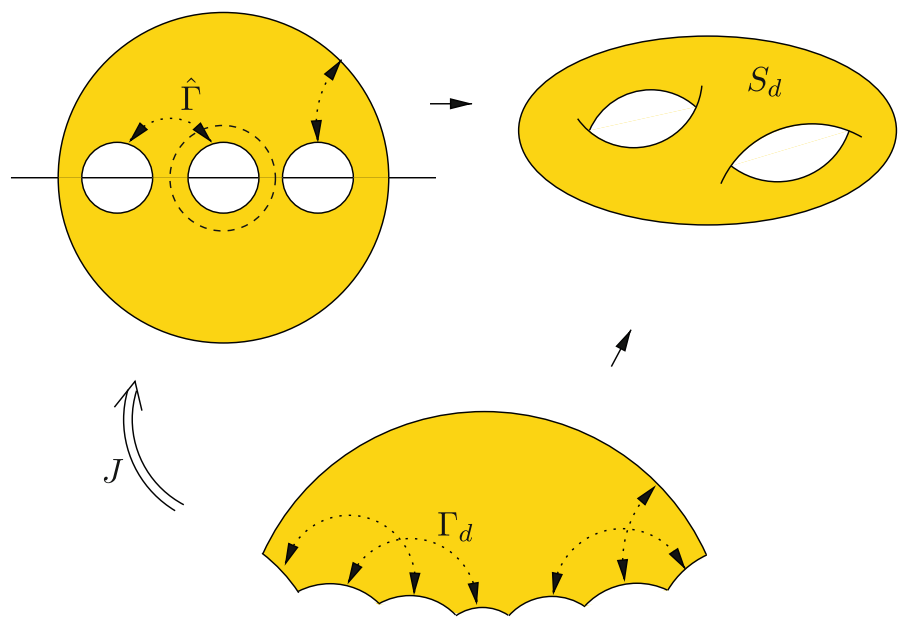

Fig. 6. The Riemann surface $S_{d}$ was originally obtained as $\left(S^{2} \backslash \Lambda(\hat{\Gamma})\right) / \hat{\Gamma}$. However, like any closed Riemann surface with $g>1$ it can also be described as $H / \Gamma_{d}$ for some $\Gamma_{d}$ for which we have drawn a fundamental domain in the bottom figure. $J$ is a locally biholomorphic map interpolating between the two descriptions. The dashed circle is a homotopically nontrivial closed curve on $S_{d}$ that can be contracted in the bulk

Since $J$ is by construction locally biholomorphic, we can use the locally defined $J^{-1}$ to pull back the metric (9) from $H$ to $S^{2} \backslash \Lambda(\Gamma)$. In Poincaré coordinates for $H^{3}$ defined in (26), the induced metric on the boundary $S^{2}$ was the flat metric $d s_{(0)}^{2}=d w d \bar{w}$ with $w=x+i \tau$. On the other hand, when we pull back the metric from $H$ using $J^{-1}$, we find a metric on this $S^{2}$ which is of the form:

$$
d s_{(0)}^{2}=\left|\frac{d J^{-1}}{d w}\right|^{2} \frac{d w d \bar{w}}{\operatorname{Im}\left(J^{-1}(w)\right)^{2}} \equiv e^{2 \sigma} d w d \bar{w} .
$$

This metric is just a Weyl rescaling of the original metric:

$$
d w d \bar{w} \mapsto e^{2 \sigma} d w d \bar{w},
$$

where we note that $\sigma$ becomes singular whenever $\operatorname{Im}\left(J^{-1}(w)\right)$ vanishes, which is precisely at the fixed point set $\Lambda(\hat{\Gamma})$ on $S^{2}$.

We may now investigate what happens to the one-point function of the stress energy tensor. Recall that in three dimensions the metric near the conformal boundary can always be put in the Fefferman-Graham form [14], $d s^{2}=\frac{d \rho^{2}}{\rho^{2}}+\frac{1}{\rho^{2}}\left(g_{(0) i j}+\rho^{2} g_{(2) i j}+\rho^{4} g_{(4) i j}\right) d x^{i} d x^{j}, \quad g_{(4) i j}=\frac{1}{4}\left(g_{(2)} g_{(0)}^{-1} g_{(2)}\right)_{i j}$,

and the one-point function of the stress energy tensor in the dual state is given by [18]

$$
\left\langle T_{i j}\right\rangle=2 g_{(2) i j}+R_{(0)} g_{(0) i j},
$$

with $R_{(0)}$ the scalar curvature of $g_{(0) i j}$ and we set $16 \pi G_{N}=1$. 
In the case at hand, starting with the bulk metric (27), we find that $d s_{(0)}^{2}=d w d \bar{w}$ and $\left\langle T_{i j}\right\rangle_{g_{(0)}}=0$. A bulk diffeomorphism that induces the Weyl rescaling in (32) has the effect of transforming the $g_{(2)}$ such that [19]

$$
\left\langle T_{w w}\right\rangle_{e^{2 \sigma} g}=\left\langle T_{w w}\right\rangle_{g}+2 \partial_{w}^{2} \sigma-2\left(\partial_{w} \sigma\right)^{2},
$$

in agreement with CFT expectations. Since in our case

$$
\sigma=\frac{1}{2} \ln \left(\partial_{w} J^{-1}\right)+\frac{1}{2} \ln \left(\overline{\partial_{w} J^{-1}}\right)-\ln \left(\frac{1}{2 i}\left(J^{-1}-\bar{J}^{-1}\right)\right),
$$

we obtain directly that

$$
\left\langle T_{w w}\right\rangle_{e^{2 \sigma} g}=\frac{\partial_{w}^{3} J^{-1}}{\partial_{w} J^{-1}}-\frac{3}{2}\left(\frac{\partial_{w}^{2} J^{-1}}{\partial_{w} J^{-1}}\right)=S\left[J^{-1}\right](w),
$$

with $S[f](w)$ the Schwarzian derivative of $f(w)$,

$$
S[f]=\frac{f^{\prime \prime \prime}}{f^{\prime}}-\frac{3}{2}\left(\frac{f^{\prime \prime}}{f^{\prime}}\right)^{2} .
$$

We therefore find that in the metric (31), the one-point function of the energy-momentum tensor is given by (37). This is already an encouraging result: we mentioned above that the bulk geometry is captured by $J$ and here we find that the same $J$ arises in the boundary energy-momentum tensor, which therefore provides the holographic encoding of the bulk geometry. However, the boundary metric (31) also depends on $J$ which is not completely intuitive. This can be avoided by using $J$ once more to pull back everything to $H$. If we use a complex coordinate $z$ on $H$, so $w=J(z)$, then we find that:

$$
\left\langle T_{z z}\right\rangle=-S[J] \quad d s_{(0)}^{2}=\frac{d z d \bar{z}}{\operatorname{Im}(z)^{2}},
$$

where we used that $\left(S\left[J^{-1}\right] \circ J\right)(d J / d z)^{2}=-S[J]$, which follows from [27]

$$
S[f \circ g]=(S[f] \circ g)(d g / d z)^{2}+S[g] .
$$

This equation may be directly verified by using the chain rule for differentiation, which in our notation is written as $(f \circ g)^{\prime}=\left(f^{\prime} \circ g\right) g^{\prime}$.

Equation (39) is the result we are after: if we describe the boundary $S_{d}$ of the handlebody as the quotient $H / \Gamma_{d}$ (corresponding to the bottom picture in Fig. 6), then the one-point function of the stress energy tensor in the constant negative curvature metric is given by minus the Schwarzian derivative of the map $J$ to $S^{2}$. If we now recall that $J$ dictates which cycles in $\Gamma_{d}$ are contractible in the bulk, namely precisely those for which $J \circ \gamma_{d}=J$, then this implies that $\left\langle T_{z z}\right\rangle$ indeed encodes the precise filling and therefore the bulk geometry.

Notice also that $S[J]$ has the right transformation properties under composition of $J$ with $S L(2, \mathbb{R})$ from the right, under which it transforms covariantly, and with $S L(2, \mathbb{C})$ from the left, under which it is invariant. These transformation properties follow from (40) and the fact that $S[f]=0$ if $f$ is a Möbius transformation [27].

Finally, let us mention that the renormalized on-shell bulk gravity action has been computed in [15] and shown to be equal to the on-shell Liouville action on $S_{d}$, computed earlier in the mathematics literature [32]. Note also that the map $J$ implicitly defines a solution to the Liouville equation. 
4.1. Bulk interpretation and relation to Teichmüller theory. We can make the holographic encoding of the spacetime a little more explicit. As mentioned above, from the bulk perspective the boundary Weyl rescaling is induced by a bulk diffeomorphism that preserves the Fefferman-Graham form of the metric but introduces a new FeffermanGraham radial coordinate $\rho^{\prime}[19,33]$. For the case at hand, the precise bulk diffeomorphism is given in $[24,30]$ and leads to the bulk metric

$$
d s^{2}=\frac{d \rho^{2}}{\rho^{2}}+\frac{\left(1+\frac{1}{4} \rho^{2}\right)^{2}}{\rho^{2}} \frac{\left|d z+\mu_{\rho} d \bar{z}\right|^{2}}{\operatorname{Im}(z)^{2}},
$$

with $z$ again a coordinate on $H$ and

$$
\mu_{\rho}(z, \bar{z})=-\frac{1}{2} \frac{\rho^{2}}{1+\frac{1}{4} \rho^{2}}(\overline{S[J](z)}) \operatorname{Im}(z)^{2},
$$

where the bar indicates complex conjugation and we dropped the primes on the new coordinates. Indeed, by expanding this metric in $\rho^{2}$ and using (34) we obtain again the result (39). It is noteworthy to mention that in the new coordinates the action of $\Gamma$ leaves slices of constant $\rho$ invariant, so its elements $\gamma$ just act as $(\rho, z) \rightarrow(\rho, \gamma(z))$ with $\gamma(z)$ given by (8).

We expect these new coordinates to become ill-defined somewhere inside the handlebody since the contractible cycles shrink to zero length at a certain point. By inspection of (41), this only happens when $|S[J](z)| \operatorname{Im}(z)^{2}>\frac{1}{2}$. This bound on the Schwarzian derivative is familiar from Teichmüller theory as it figures prominently in the Ahlfors-Weil theorem concerning a local inverse of Bers' embedding of Teichmüller spaces [27] in the space of holomorphic quadratic differentials. The physical relevance of the bound is the following. When this bound is nowhere satisfied the coordinate system is nonsingular all the way to $\rho \rightarrow \infty$, where we recover another asymptotically AdS region. We then do not describe a wormhole but rather a spacetime with two disconnected boundaries which are simultaneously uniformized in the boundary $S^{2}$, as expected from Teichmüller theory. These do not correspond to wormholes and we refer to [34] for more information as well as open questions regarding these spaces. For a handlebody there are no other asymptotic regions and we may therefore assume on physical grounds that the bound is everywhere satisfied. In that case, the coordinate system becomes degenerate at a surface given by

$$
\rho^{2}=\rho_{c}^{2} \equiv \frac{1}{|S[J]| \operatorname{Im}(z)^{2}-\frac{1}{2}} .
$$

At the surface $\rho=\rho_{c}$, the metric is everywhere degenerate since $\left|\mu_{\rho_{c}}\right|=1$. We then describe a point in the boundary of Teichmüller's compactification of the Teichmüller space [27]. It would be interesting to verify explicitly that the contractible cycles are indeed the degenerate cycles on this surface.

4.2. Non-handlebodies. The discussion so far was about Euclidean handlebodies, but these are not the only 3-manifolds that have a genus $g$ Riemann surface as their conformal boundary. We briefly discuss an example of such non-handlebody spacetimes in this subsection. ${ }^{5}$ A simple example can be constructed from the spacetimes described

\footnotetext{
5 We thank Alex Maloney for discussions about the material in this subsection.
} 
in [34]. These are obtained by starting from $H^{3}$ written in hyperbolic slicing and quotienting the boundary by a discrete subgroup $\Gamma$ of $H$ to obtain a compact, finite volume, genus $g>1$ surface, $\Sigma_{g}$. This yields the metric with two boundaries,

$$
d s^{2}=d r^{2}+\cosh ^{2}(r) d s_{\Sigma_{g}}^{2}
$$

where $r \in(-\infty, \infty)$ and

$$
d s_{\Sigma_{g}}^{2}=\frac{d z d \bar{z}}{\operatorname{Im}(z)^{2}}
$$

is the constant negative curvature metric on $\Sigma_{g}$ which has scalar curvature $R=-2$. To produce a manifold with a single boundary, one may try to quotient by $r \rightarrow-r$. This procedure however introduces a singularity at $r=0$. The singularity can be avoided if the surface $\Sigma_{g}$ has a fixed point free involution $I$, since then we can combine $r \rightarrow-r$ together with the action of $I$ to obtain a smooth hyperbolic 3-manifold with conformal boundary the Riemann surface $\Sigma_{g}$. Such involutions are discussed, for example, in [35]. In this case the singularity at $r=0$ is replaced by the smooth Riemann surface $\Sigma_{g} / I$. The resulting 3-manifold is a quotient of $\mathrm{H}^{3}$ which has no contractible cycles so it is not a handlebody.

This 3-manifold has the same conformal boundary as the handlebody build from $\Sigma_{d}$ but it has a different expectation value of the energy momentum tensor. Changing variable, $\rho=2 e^{-r}$, the metric becomes of the form (33)) with:

$$
g_{(0) i j}=2 g_{(2) i j}=d s_{\Sigma_{g}}^{2} .
$$

We may then use (34) to obtain that:

$$
\left\langle T_{i j}\right\rangle=-g_{(0) i j}
$$

We see that the one-point function of the energy-momentum tensor is notably different from that of a handlebody. However, we also observe that any involution that 'ends' the spacetime at $r=0$ (with or without fixed points, orientation-reversing or orientationpreserving) results in the same one-point function, so the holographic one-point function of $T_{i j}$ does not seem able to distinguish these geometries.

This is an interesting subtlety of the Euclidean dictionary due to global issues. Let us recall why we expect that locally, in the Euclidean setup, $g_{(0) i j}$ and $\left\langle T_{i j}\right\rangle$ uniquely fix a bulk solution (in any dimension). Intuitively, this is because the bulk equations of motion are second order differential equations and $g_{(0) i j}$ and $\left\langle T_{i j}\right\rangle$ provide the correct initial data. One can indeed show rigorously that given this data there exists a unique bulk solution in a thickening of the conformal boundary, see [36] and references therein. Furthermore, one can show that $\left(g_{(0) i j}, T_{i j}\right)$ are coordinates in the covariant phase space of the theory [37] and thus each such pair specifies a solution. In the case at hand this data indeed produces a unique metric for $r>0$, but the way the spacetime is capped off at $r=0$ depends on the fixed point free involution used. One can presumably distinguish the different spacetimes by using higher point functions and non-local observables, such as the expectation values of Wilson loops, i.e. minimal surfaces that end at a loop in the conformal boundary of the 3-manifold. It would be interesting to verify this explicitly. 


\section{Holographic Interpretation of Lorentzian Wormholes}

We now move to discuss the holographic interpretation of the Lorentzian wormholes. We start by demonstrating in the next subsection that a naive adaptation of the analysis of the previous section leads to incomplete results where, in contrast with the Euclidean results, the spacetime geometry does not seem to be captured by the dual field theory on the Lorentzian side. This is then resolved using the real-time gauge/gravity prescription of $[4,5]$.

5.1. Naive computation. As we mentioned earlier, the metric in the outer regions can always be cast in the BTZ form (20). When using a new coordinate $\rho$ defined via

$$
r=\frac{\frac{M}{4} \rho^{2}+1}{\rho},
$$

the metric takes the form in (33) with

$$
g_{(0) i j}=\eta_{i j}, \quad g_{(2) i j}=\frac{M}{2} \delta_{i j}, \quad g_{(4) i j}=\frac{M^{2}}{16} \eta_{i j} .
$$

The one-point function of the stress energy tensor in the dual state can be computed from (34) yielding,

$$
\left\langle T_{i j}\right\rangle=M \delta_{i j} .
$$

On the other $(m-1)$ conformal boundaries, we obtain similar one-point functions (with different values of $M$ ) and all the other one-point functions vanish. This is problematic, since we obtain no information whatsoever about the inner part of the geometry and the Lorentzian one-point functions therefore seem to be insufficient to reconstruct the wormhole spacetime. The holographic encoding of the spacetime appears to fail, which would contradict standard expectations from the gauge/gravity duality. This apparent contradiction comes from the fact that we have not taken into account the holographic interpretation of Cauchy data. This can be done using the real-time gauge/gravity prescription of $[4,5]$, which we review in the next subsection.

5.2. Lorentzian gauge/gravity prescription. In this subsection, we prepare for the discussion below by reviewing some known facts about Lorentzian quantum field theory. Afterwards, we show how one may use these facts to obtain a consistent real-time prescription for the gauge/gravity duality. We then return to the wormholes in Subsect. 5.3.

5.2.1. States in field theory. The prescription in $[4,5]$ is based on the fact that any Lorentzian field theory path integral requires a specification of the initial and final states as well. Such a state $|\Psi\rangle$ may be specified via path integrals on a Euclidean space $Y$ with a boundary and possible operator insertions away from this boundary. If we want to compute, say, $\langle\Psi|\mathcal{O}(t)| \Psi\rangle=\left\langle\Psi\left|e^{i H t} \mathcal{O} e^{-i H t}\right| \Psi\right\rangle$, we continue to path integrate along a Lorentzian segment with length $t$ that is glued to the boundary of the Euclidean space, then insert the operator, and finally go back in time for a period $t$ before we attach a second copy of the Euclidean space. For Euclidean spaces which are topologically $\mathbb{R} \times X$ with $X$ a real space and $\mathbb{R}$ representing Euclidean time, the overall field theory 


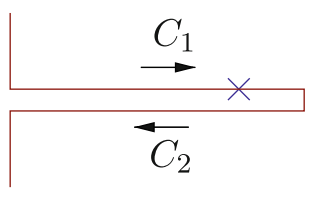

Fig. 7. A contour in the complex time plane; the cross signifies the operator insertion

background manifold corresponds to a contour in the complex boundary time plane of the form sketched in Fig. 7 times a real space. Notice that extending the contour beyond the point $t$, say to a point $T>t$, amounts to an extra insertion of $e^{i H(T-t)} e^{-i H(T-t)}=\mathbf{1}$ which does not affect the correlation function. A similar story holds for higher-point correlation functions, but in those cases an operator ordering has to be specified. Although the contour may often be deformed to a simpler version, we emphasize that a procedure like the above is always necessary for Lorentzian quantum field theory.

5.2.2. Translation to gravity. In the Lorentzian gauge/gravity prescription of $[4,5]$, one incorporates the Euclidean segments for the path integral into the holographic description and 'fills' them with a bulk solution as well. For example, to the contour of Fig. 7 may correspond a bulk manifold consisting of two Lorentzian and two Euclidean segments. These segments are then glued to each other along spacelike hypersurfaces that should end on the corners of the boundary contour. The behavior of the fields at these hypersurfaces is then determined using matching conditions. These guarantee the $C^{1}$ continuity of the fields. More precisely, for the metric one imposes continuity of the induced metric $h_{A B}$ and the extrinsic curvature $K_{A B}$ with a factor of $i$ :

$$
{ }^{L} h_{A B}={ }^{E} h_{A B}, \quad{ }^{L} K_{A B}=-i{ }^{E} K_{A B},
$$

with the superscript indicating the Lorentzian or the Euclidean side and the extrinsic curvature on either side is defined using the outward pointing unit normal. There is also a corner matching condition, ${ }^{6}$ which is defined at the intersection between $S$ and the conformal (radial) boundary. It dictates that the inner product between the unit normal to $S$, denoted as $n_{\mu}$, and the unit normal to the radial boundary, written as $\hat{n}_{\mu}$, is continuous across the boundary (up to appropriate factors of $i$ ). For a Lorentzian-Euclidean gluing, using outward pointing unit normals, it becomes:

$$
\left.L_{\left(\hat{n}^{\mu}\right.} n_{\mu}\right)=i^{E}\left(\hat{n}^{\mu} n_{\mu}\right) .
$$

As discussed in [5], all the matching conditions arise naturally from a saddle-point approximation. Although they are equivalent to analytic continuation in many simple cases, they do not rely on a globally defined time coordinate and are therefore more generally applicable.

This construction is an essential ingredient in the Lorentzian gauge/gravity dictionary. For example, it allows us to understand precisely how changing the initial and final states modifies the Lorentzian spacetime, gives the correct initial and final conditions for the bulk-boundary and bulk-bulk propagators, and also cancels surface terms from timelike infinity in the on-shell action, which would otherwise lead to additional infinities. Furthermore, the boundary correlators directly come in the in-in form as in quantum field theory.

\footnotetext{
6 It is likely that this condition follows from the matching for the induced metric and the extrinsic curvature in (51) but in the absence of a general proof we treat it as an additional matching condition.
} 


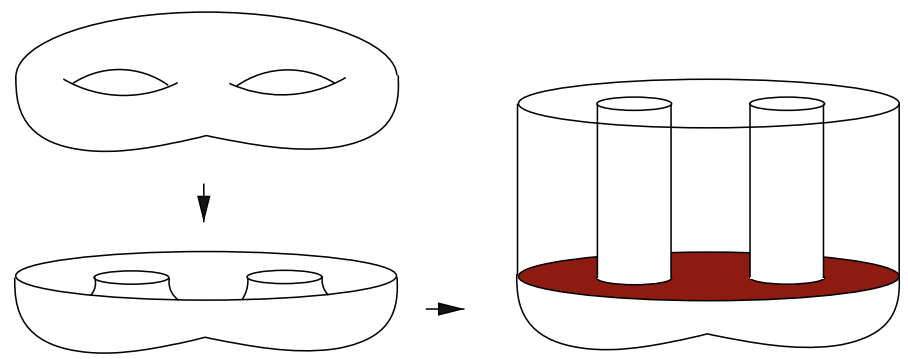

Fig. 8. We take half of a genus two surface and attach Lorentzian cylinders to the boundary. This boundary manifold can be filled in with half an Euclidean handlebody plus a Lorentzian wormhole with spatial topology of a pair of pants. On the right, we shaded the matching surface between the Euclidean and the Lorentzian segment. It indeed has three boundaries and no handles

5.3. Gauge/gravity duality for Lorentzian wormholes. Let us now apply the construction outlined in the previous subsection to the wormholes. To this end, we have to cut off the wormhole along some spatial bulk hypersurface and find a Euclidean space that we may glue to this hypersurface such that the matching conditions are satisfied.

Of course the field theory contour also has a backward-going segment and a final state. To fill this in, we have to cut off the wormhole along some final time slice as well and glue a second Lorentzian and Euclidean segment to this final surface. These second copies can be taken to be identical to the first ones, which correspond to taking the final and the initial state to be just the same. In [5], we performed this procedure for the eternal BTZ black hole. As long as we do not switch on any perturbations, we may take the second Lorentzian and Euclidean segment to be completely identical to the first one. This also means that the matching conditions are trivially satisfied along the final gluing surface, so these do not have to be investigated separately. Therefore, it will be sufficient to focus on a single Euclidean-Lorentzian gluing below.

A candidate for the Euclidean space is half of the Euclidean handlebody $M_{e}$ that we obtained in Sect. 3. (It is not however the only candidate, as we will explicitly demonstrate in Subsect. 5.4.) Indeed, we may cut this handlebody and the wormhole spacetime in two halves along the surface $S$ and glue them together along $S$. In the case $S$ is a pair of pants (a surface of genus zero with three circular boundaries, so $g=0$ and $m=3$ ), the procedure is sketched in Fig. 8 and the filling of the full field theory contour, including the backward-going segment, is sketched in Fig. 9. Let us now verify that the matching conditions are satisfied at the shaded matching surface in Fig. 8. On both sides, the induced metric is locally just the unique negative curvature metric on $S$ described as $H / \Gamma$, so it is the same metric indeed. Also, the extrinsic curvature vanishes completely on both sides because of the $\mathbb{Z}_{2}$ time-reversal symmetry. Therefore, the first and second matching conditions are satisfied indeed. Finally, the extra corner matching discussed in [5] is also satisfied: in our case $S$ intersects the conformal boundary orthogonally (again because of the $\mathbb{Z}_{2}$ symmetry) and the inner product $n^{\mu} \hat{n}_{\mu}$ thus vanishes both for $M$ and for $M_{e}$. However, there is still a subtlety with the boundary metric which we now discuss.

If we use the BTZ coordinate system on the Lorentzian side, then the boundary metric on this side is flat. The boundary metric on the Euclidean side, however, can never be globally flat because $S_{d}$ has negative Euler number. On the other hand, to match $M$ and $M_{e}$, we should also take the boundary metric to be smooth (in the sense specified in [5]). This can be done by Weyl rescaling the Lorentzian boundary metric to a metric of 


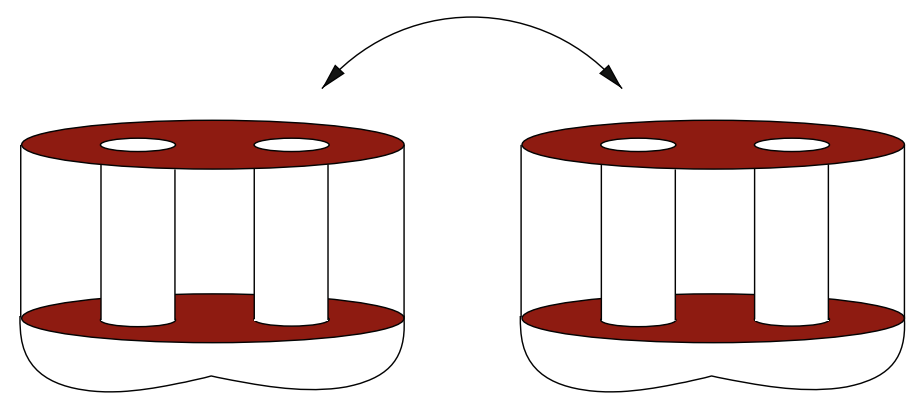

Fig. 9. Analogous to Fig. 7, the full field theory contour has a forward- and a backward-going segment and two Euclidean segments to specify the initial and final state. Similarly, the full bulk spacetime consists of four segments as well. They should be glued along the matching surfaces which we shaded in this picture

constant negative curvature, as we discuss below. The boundary metric is then smooth across the corner and the discrepancy between the boundary metrics on either side is removed. ${ }^{7}$

5.3.1. Matching Euclidean and Lorentzian wormholes and $\left\langle T_{i j}\right\rangle$. We now discuss the consequences of the continuity of the boundary metric across the matching surface. As described above we match the initial $U=0$ surface of the Lorentzian wormhole to half of the Euclidean handlebody. On the boundary of the spacetime, the Lorentzian cylinders are glued to the boundary of the Euclidean handlebody along the $m$ circles that form the boundary of the $U=0$ Riemann surface. These $m$ circles lift to segments of the great circle given by $\tau=0$ in the Poincaré coordinates (26) on the boundary $S^{2}$ of $H^{3}$. Let us now focus on one of the $m$ circles. After conjugation, we can always ensure that it lifts to the half-line $l$ given by:

$$
l: x>0, \quad \tau=0
$$

on the $S^{2}$. Its projection down to $S_{d}$ is then given via the identification

$$
w \sim \lambda w
$$

with $w=x+i \tau$ and for some positive real $\lambda \neq 1$. The relevant part of the fundamental domain is then sketched in Fig. 10.

To find the boundary metric of constant curvature on this surface we again have to pass from the description of $S_{d}$ as quotient of $\left(S^{2} \backslash \Lambda(\hat{\Gamma})\right)$ to that of a quotient of $H$, for which we defined the map $J$ in Sect. 4. Using $J^{-1}$, we now map the half-line (53) to $H$, where we use the coordinate $z$. Although $J^{-1}$ is multi-valued, we will need only one of the images of $l$ in $H$. We can again use conjugation freedom to make sure that the image under consideration is the half-line:

$$
l^{\prime}: \operatorname{Re}(z)=0 .
$$

In $H$, the identification (54) becomes an isometry of $S L(2, \mathbb{R})$ that leaves $l^{\prime}$ invariant. Such an isometry is necessarily of the form:

$$
z \sim \mu z
$$

7 Another possibility would be to Weyl rescale the metric on the Euclidean side such that it is flat in the vicinity of the gluing circles. Although the gluing is then smooth, the Euclidean boundary metric can then no longer be analytic. 


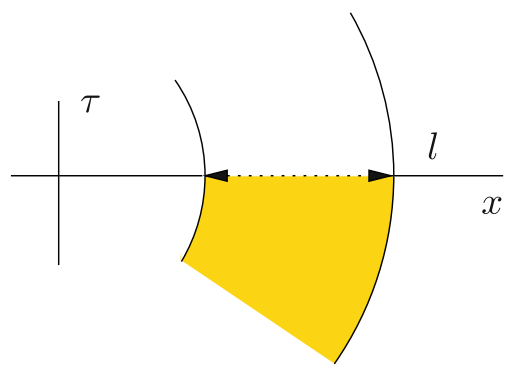

Fig. 10. Part of a fundamental domain of $S_{d}$ on $S^{2}$. We will eventually replace the part with $\tau>0$ with a Lorentzian wormhole. The single identification is given by $w \sim \lambda w$ and the line $l$ is the entire positive $x$-axis

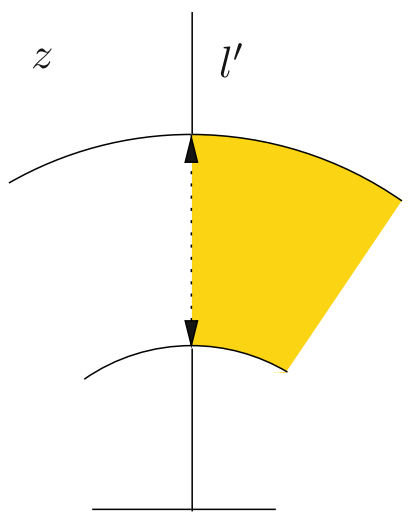

Fig. 11. Under the locally defined map $J^{-1}$ the domain in Fig. 10 maps to the sketched domain in $H$, where we use a coordinate $z$. The identification is given by $z \sim \mu z$. The line $l^{\prime}$ is the positive imaginary axis. We will replace the part $\operatorname{Re}(z)<0$ with a Lorentzian wormhole

for some real $\mu \neq 1$ given implicitly by

$$
J(\mu z)=\lambda J(z) .
$$

The construction in $H$ is sketched in Fig. 11. Notice that $J(z)$ is an analytic map from the imaginary axis to the (positive) real axis, that is

$$
\overline{J(i y)}=J(i y), \quad y>0 .
$$

Notice also that the $\mathbb{Z}_{2}$ symmetry $w \leftrightarrow \bar{w}$ maps under $J^{-1}$ to reflection in the imaginary axis, that is $z \leftrightarrow-\bar{z}$. (Again, as $J^{-1}$ is multi-valued, it maps the original $\mathbb{Z}_{2}$ to many other reflections in $H$ as well, but we do not need them here.)

We now ready to attach a Lorentzian cylinder to the boundary. The procedure is sketched in Fig. 12. On $H$, this means that we cut away the half given by $\operatorname{Re}(z)<0$ and attach the universal covering of a Lorentzian cylinder to the gluing line $\operatorname{Re}(z)=0$. In the bulk, we can use the metric (41) with the matching surface given by $\operatorname{Re}(z)=0$, at least up to the point $\rho=\rho_{c}$. We now need to find a Lorentzian bulk metric that satisfies the matching conditions of [5] when glued to this surface.

Both in the bulk and on the boundary, it is straightforward to obtain the explicit matching Lorentzian metric by analytic continuation. We first introduce a coordinate 

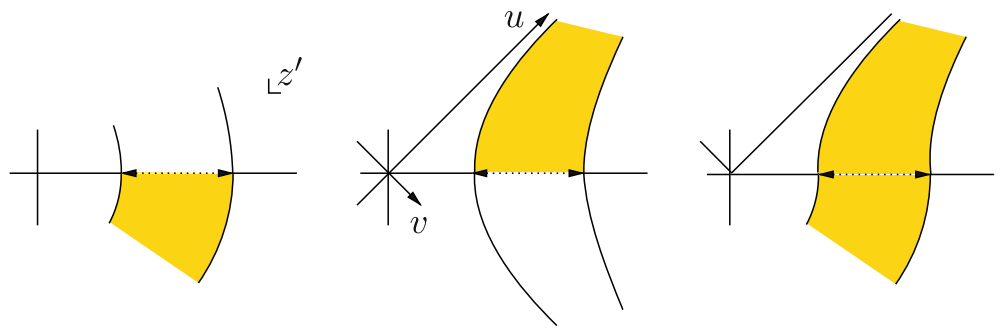

Fig. 12. On the left, the Euclidean boundary geometry in the coordinate $z^{\prime}$. In the center figure we sketched the Lorentzian boundary geometry and on the right the glued-together geometry

$z^{\prime}=-i z$. In the $z^{\prime}$ plane Fig. 11 is rotated clockwise by 90 degrees which slightly simplifies the matching below. In the coordinate $z^{\prime}$ the metric (41) becomes:

$$
d s^{2}=\frac{d \rho^{2}}{\rho^{2}}+\frac{\left(1+\frac{1}{4} \rho^{2}\right)^{2}}{\rho^{2}} \frac{\left|d z^{\prime}+\mu_{\rho} d \bar{z}^{\prime}\right|^{2}}{\operatorname{Re}\left(z^{\prime}\right)^{2}},
$$

where now

$$
\mu_{\rho}\left(z^{\prime}, \bar{z}^{\prime}\right)=\frac{1}{2} \frac{\rho^{2}}{1+\frac{1}{4} \rho^{2}}\left(\overline{S[\tilde{J}]\left(z^{\prime}\right)}\right) \operatorname{Re}\left(z^{\prime}\right)^{2}, \quad \tilde{J}\left(z^{\prime}\right)=J\left(i z^{\prime}\right),
$$

where we used that $S[J]\left(i z^{\prime}\right)=-S[\tilde{J}]\left(z^{\prime}\right)$, which follows from (40). The gluing takes place along the half-line $\operatorname{Im}\left(z^{\prime}\right)=0, \operatorname{Re}\left(z^{\prime}\right)>0$. We then replace $z^{\prime} \rightarrow u$ and $\bar{z}^{\prime} \rightarrow v$ to find the Lorentzian bulk metric:

$$
d s^{2}=\frac{d \rho^{2}}{\rho^{2}}+\frac{\left(1+\frac{1}{4} \rho^{2}\right)^{2}}{\rho^{2}} \frac{\left(d u+\mu_{\rho}(v) d v\right)\left(d v+\mu_{\rho}(u) d u\right)}{\frac{1}{4}(u+v)^{2}},
$$

with

$$
\mu_{\rho}(u)=\frac{1}{8} \frac{\rho^{2}}{1+\frac{1}{4} \rho^{2}}(S[\tilde{J}](u))(u+v)^{2},
$$

and a similar expression with $u \rightarrow v$. Note that $\tilde{J}(x)$ is real-analytic for $x>0$ and monotonic, so $S[\tilde{J}](x)$ is real-analytic too. This Lorentzian metric is thus real and covers the bulk spacetime up to $\rho=\rho_{c}$. Since $z^{\prime} \sim \mu z^{\prime}$, the periodicity on the Lorentzian side is $(u, v) \sim \mu(u, v)$. The point $(u, v)=(0,0)$ on the boundary is a fixed point of this identification and therefore we need to exclude the forward lightcone emanating from this point from the spacetime (the backward lightcone is already replaced by the Euclidean geometry). Since we also demanded $\operatorname{Re}\left(z^{\prime}\right)>0$, so $u+v>0$, we need only the part of the Lorentzian boundary with $u>0$ and $v>0$.

On the boundary we find the metric:

$$
d s_{(0)}^{2}=\frac{d u d v}{\frac{1}{4}(u+v)^{2}}
$$

which has scalar curvature $R_{(0)}=-2$. Using once more (34) we obtain for the one-point functions:

$$
\left\langle T_{u u}\right\rangle=-S[\tilde{J}](u), \quad\left\langle T_{v v}\right\rangle=-S[\tilde{J}](v), \quad\left\langle T_{u v}\right\rangle=\frac{-1}{8(u+v)^{2}} .
$$




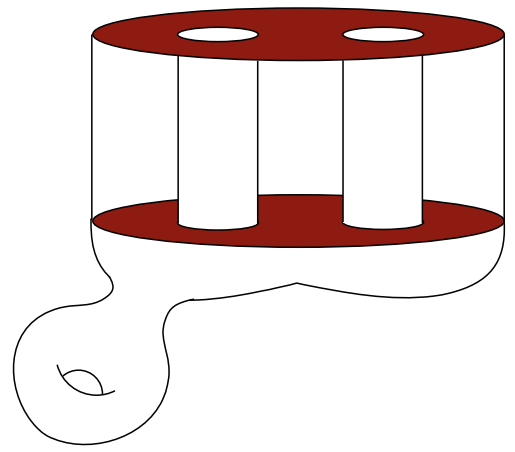

Fig. 13. Adding a handle as indicated does not change the properties of the gluing surface or the Lorentzian spacetime

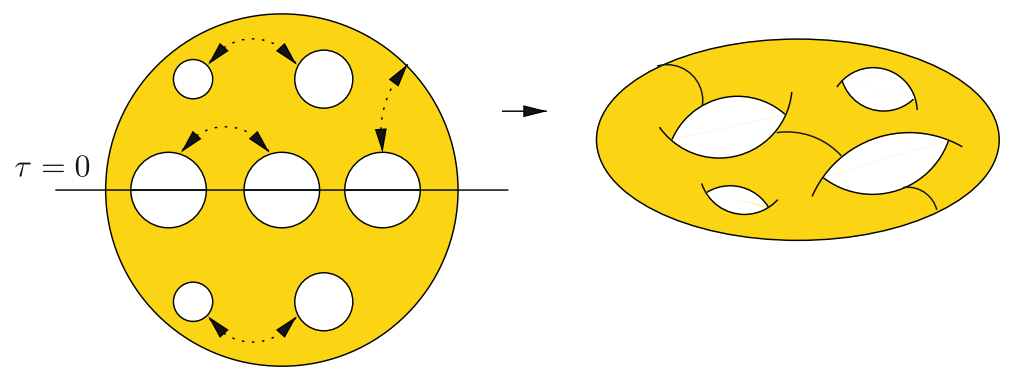

Fig. 14. As indicated on the left, one may add generators to the Schottky group $\hat{\Gamma}$ without breaking the $\mathbb{Z}_{2}$ symmetry. The resulting surface has two extra handles: one for the half corresponding to the initial state and another one for the final state

Notice that one expects that $T_{i}^{i}=\frac{c}{24 \pi} R_{(0)}$ and we obtained here $T_{i}^{i}=-2$. Reinstating the factors of $16 \pi G_{N}$, we find $c=24 \pi /\left(16 \pi G_{N}\right)=3 /\left(2 G_{N}\right)$ which is indeed the correct central charge.

Equation (64) is the main result of this section and demonstrates that the Lorentzian one-point function of the stress energy tensor as obtained from the metric (61) does contain information about the dual geometry that is hidden behind the horizons.

5.4. More fillings. In the previous section we glued a particular handlebody to the Lorentzian wormhole. There exist a variety of handlebodies $\left\{M_{e}\right\}$ that all have a hypersurface $S$ where the matching conditions are satisfied as we discuss now.

In particular, one may attach an extra filled handle to $M_{e}$ somewhere away from the matching surface to obtain a manifold $M_{e}^{\prime}$ with conformal boundary $\Sigma$ with $\Sigma \neq S_{d}$. An example of this is sketched in Fig. 13. This procedure does not change any properties like the induced metric or the extrinsic curvature of the matching surface. Geometrically, this can be seen by going to the universal covering: one may add generators to $\hat{\Gamma}$ to obtain a group $\hat{\Gamma}^{\prime}$ and as long as $M_{e}^{\prime}=H^{3} / \hat{\Gamma}^{\prime}$ has $S$ as a surface of $\mathbb{Z}_{2}$ symmetry we may slice open $M_{e}^{\prime}$ along this surface and glue the Lorentzian wormhole $M_{l}=\widehat{\operatorname{AdS}}_{3} / \hat{\Gamma}$ to it. For the boundary surface this procedure is sketched in Fig. 14. In the figure we represented the addition of two generators to $\hat{\Gamma}$ by cutting four circles out of the fundamental domain that are pairwise identified, all done in such a way that the original $\mathbb{Z}_{2}$ 
symmetry remains intact. Although we have not sketched it here, this procedure directly extends to the entire three-dimensional space: the $\mathbb{Z}_{2}$ symmetry is also present for the new handlebody and $S$ is again the invariant surface given by $\tau=0$.

We conclude that we can glue to the Lorentzian wormholes also half of $M_{e}^{\prime}$. A similar analysis as in the previous section establishes that the 1-point functions captures the fact that the initial state is different than the one corresponding to $M_{e}$.

5.5. State dual to wormholes. Let us now discuss what our results imply about the QFT state dual to the wormholes. Since there are $m$ boundaries, the Hilbert space consists of a tensor product of $m$ Hilbert spaces, one for each boundary component. From the fact that the wormholes are manifolds that interpolate between the $m$ segments, we expect to find nonzero correlations between the $m$ boundaries and the initial state to be an entangled state. Indeed, this is precisely what we find. To see this, suppose the initial state is separable, namely of a product form $\left|\alpha_{1}\right\rangle \otimes \cdots \otimes\left|\alpha_{m}\right\rangle$. Then the 1-point functions would necessarily take a factorizable form. More precisely, suppose the state was separable and consider the insertion of a stress energy tensor in, say, the first boundary component,

$$
\begin{aligned}
\left\langle T_{i j}\left(x_{1}\right)\right\rangle & =\left\langle\alpha_{1}\left|\otimes \cdots \otimes\left\langle\alpha_{m}\left|T_{i j}\left(x_{1}\right)\right| \alpha_{1}\right\rangle \otimes \cdots \otimes\right| \alpha_{m}\right\rangle \\
& =\left\langle\alpha_{1}\left|T_{i j}\left(x_{1}\right)\right| \alpha_{1}\right\rangle \prod_{k=2}^{2} \|\left|\alpha_{k}\right\rangle \|^{2} .
\end{aligned}
$$

Now the naive 1-point function in (50) would support the view that the state is separable. Namely, in that case we could naively say that the state $\left|\alpha_{1}\right\rangle$ in the first copy depends only on the corresponding mass parameter $M_{1}$ and not on the other variables that determine the spacetime. This would lead to one- and higher-point functions of the energy momentum tensor in the first copy which up to an overall factor only depend on $M_{1}$. The one-point functions that we got, however, in (64) are not of that form, as the Schwarzian $S[\tilde{J}]$ does not have such a factorizable form and does contain all the variables that determine the spacetime.

Another check on the non-separability of the state is provided by the computation of a two-point function. An argument analogous to the one above implies that if the state is separable then the 2-point function would have a factorizable form. We illustrate that this is not the case in the next subsection.

A natural guess for the dual state is that it is the state obtained by an Euclidean path integral over a Riemann surface $\Sigma$ with $m$ circular boundaries. According to the reasoning of $[11,38]$, this surface $\Sigma$ can be taken to be precisely the conformal boundary of one half of the Euclidean manifold $M_{e}$. This can be $\Sigma=S$, with $S$ the surface of time reversal symmetry of the wormhole spacetime, for the case of the handlebodies of Sect. 3, or $\Sigma \neq S$ if the initial state is that of the previous subsection.

If we now trace over all components but one, all wormholes with $m>1$ can be thought of as having been associated with a mixed state in the remaining copy and this explains the presence of horizons. The $m=1$ case is special in that we only have a single copy of the CFT so there are no copies to trace out. Nevertheless results for the 1- and 2-point functions indicate that there is an entanglement between the outer region and the region behind the horizon. These spacetimes were also analyzed in [39] which suggested that the dual state is in some respects similar to a thermal state. We leave a better understanding of this case for future work. 
5.6. 2-point functions. We discuss in this subsection the computation of the 2-point function for a scalar operator $\mathcal{O}$ of dimension $\Delta$. In the bulk it suffices to consider a free massive scalar field, as interaction terms contribute only to higher point functions. We glue an Euclidean handlebody at $t=0$ and take the initial and final states to be the same. It follows from an analysis along the lines of [5] that the different real-time correlators (time-ordered, Wightman, etc.) are obtained by suitable analytic continuations of the Euclidean correlator in the handlebody geometry.

The two-point function of a scalar operator on the Euclidean plane is uniquely fixed by conformal invariance and takes the form:

$$
\langle\mathcal{O}(\tau, x) \mathcal{O}(\hat{\tau}, \hat{x})\rangle=\frac{1}{\left[(\tau-\hat{\tau})^{2}+(x-\hat{x})^{2}\right]^{\Delta}},
$$

where we normalized the operators so that the coefficient in the numerator equals one. For the handlebody, we have to sum over the elements of the Schottky group $\hat{\Gamma}$, whose elements $\gamma$ act as Möbius transformations on the boundary,

$$
\gamma: \omega=x+i \tau \rightarrow \frac{a(x+i \tau)+b}{c(x+i \tau)+d}
$$

with real $a, b, c, d$ and $a d-b c=1$. This can also be written as

$$
\gamma:(\tau, x) \rightarrow\left(\gamma_{\tau}, \gamma_{x}\right) \equiv \frac{1}{(c x+d)^{2}+c^{2} \tau^{2}}\left(\tau,(a x+b)(c x+d)+a c \tau^{2}\right) .
$$

Using the complex coordinate $w$ on the boundary $S^{2}$ of $H^{3}$, we obtain

$$
\left\langle\mathcal{O}(w, \bar{w}) \mathcal{O}\left(w_{1}, \bar{w}_{1}\right)\right\rangle=\sum_{\gamma \in \hat{\Gamma}} \frac{1}{|c w+d|^{2 \Delta}\left|\gamma_{w}-w_{1}\right|^{2 \Delta}}
$$

where the boundary metric is locally $d w d \bar{w}$. We then Weyl transform to the metric (31) which is globally well-defined to find

$$
\left\langle\mathcal{O}(w, \bar{w}) \mathcal{O}\left(w_{1}, \bar{w}_{1}\right)\right\rangle=\sum_{\gamma \in \hat{\Gamma}} \frac{e^{-\Delta \sigma(w, \bar{w})} e^{-\Delta \sigma\left(w_{1}, \bar{w}_{1}\right)}}{|c w+d|^{2 \Delta}\left|\gamma_{w}-w_{1}\right|^{2 \Delta}}, \quad d s^{2}=e^{2 \sigma(w, \bar{w})} d w d \bar{w},
$$

with

$$
e^{2 \sigma(w, \bar{w})}=\left|\frac{d J^{-1}}{d w}\right|^{2} \frac{1}{\operatorname{Im}\left(J^{-1}(w)\right)^{2}} .
$$

We can now pull back to $H$, using $z=J^{-1}(w)$, to obtain

$$
\left\langle\mathcal{O}(z, \bar{z}) \mathcal{O}\left(z_{1}, \bar{z}_{1}\right)\right\rangle=\sum_{\gamma \in \hat{\Gamma}} \frac{\left|J^{\prime}(z) J^{\prime}\left(z_{1}\right)\right|^{\Delta}\left(\operatorname{Im}(z) \operatorname{Im}\left(z_{1}\right)\right)^{\Delta}}{|c J(z)+d|^{2 \Delta}\left|\gamma(J(z))-J\left(z_{1}\right)\right|^{2 \Delta}}, \quad d s^{2}=\frac{d z d \bar{z}}{\operatorname{Im}^{2}(z)} .
$$

As before, we may assume the covering groups are such that $J(\lambda z)=\mu J(z)$. We then again introduce the coordinate $z^{\prime}=-i z$ and the map $\tilde{J}\left(z^{\prime}\right)=J\left(i z^{\prime}\right)=J(z)$, replace 
$z^{\prime} \rightarrow u$ and $\bar{z}^{\prime} \rightarrow v$ to obtain the Lorentzian metric. We recall that in this case $\tilde{J}(x)$ is real-analytic for real positive $x$. More precisely, following the steps in [5], one finds that the time-ordered correlator is obtained by replacing $z \rightarrow u-i \epsilon u$ and $\bar{z} \rightarrow v+i \epsilon v$, where the $i \epsilon$ insertions push the singularity everywhere away from the real-time contour. To avoid clutter we will however not write the $i \epsilon$ insertions explicitly below. The final answer is then

$$
\begin{aligned}
& \left\langle T \mathcal{O}(u, v) \mathcal{O}\left(u_{1}, v_{1}\right)\right\rangle \\
& =\sum_{\gamma \in \hat{\Gamma}} \frac{2^{-2 \Delta}(u+v)^{\Delta}\left(u_{1}+v_{1}\right)^{\Delta}\left[\tilde{J}^{\prime}(u) \tilde{J}^{\prime}(v) \tilde{J}^{\prime}\left(u_{1}\right) \tilde{J}^{\prime}\left(v_{1}\right)\right]^{\Delta / 2}}{(c \tilde{J}(u)+d)^{\Delta}(c \tilde{J}(v)+d)^{\Delta}\left(\gamma(\tilde{J}(u))-\tilde{J}\left(u_{1}\right)\right)^{\Delta}\left(\gamma(\tilde{J}(v))-\tilde{J}\left(v_{1}\right)\right)^{\Delta}}
\end{aligned}
$$

in the metric

$$
d s^{2}=\frac{4 d u d v}{(u+v)^{2}} .
$$

This 2-point function does not take a factorizable form supporting the view that the dual state is entangled, as anticipated. We would like to note however that at late times the correlator is dominated by the BTZ elements in $\hat{\Gamma}$. More precisely, if one defines coordinates $^{8} u=\exp (x+t), v=\exp (x-t)$, then in the limit $t, t_{1} \rightarrow \infty$ with $\left(t-t_{1}\right)$ fixed, all terms in (73) go to zero, except the ones with either $b=0$ or $c=0$. These are precisely the elements associated with the BTZ black hole.

\section{Remarks}

In this section we discuss some general remarks concerning the wormhole spacetimes.

6.1. Other bulk spacetimes. The question we addressed in this paper is what is the holographic interpretation of any given wormhole spacetime. One can also ask: given a geometry at infinity, how many different bulk spacetimes can one have? In general, all such saddle points contribute and should be taken into account, although typically one of the saddle points dominates at large $N$ at any given regime. A well-known example is that associated with the Hawking-Page transition $[3,40]$. In that case the boundary is $S^{1} \times S^{d-1}$ and there are two possible (Euclidean) bulk manifolds corresponding to making contractible in the interior either $S^{1}$ or $S^{d-1}$, namely the Euclidean Schwarzschild AdS solution and thermal AdS. This question is usually addressed in Euclidean signature, but it is clearly also relevant in Lorentzian signature. In this context the question is now: given the conformal boundary of the complete Euclidean and Lorentzian pieces how many different bulk manifolds can one have?

Naively, one might think that for every Euclidean solution there would be a corresponding Lorentzian plus Euclidean solution, but this turns out not to be the case. This can be demonstrated with the case where the conformal boundary is a torus, $S^{1} \times S^{1}$. As in the higher dimensional case, there are two solutions that correspond to either the first or the second circle being contractible in the interior (which correspond to thermal AdS and Euclidean BTZ), but there are now new possibilities obtained by considering a contractible cycle that is a linear combination of the above cycles [41]. These solutions

\footnotetext{
${ }^{8}$ In these coordinates the Lorentzian cylinder is $(t, x) \sim(t, x+\log \lambda)$.
} 


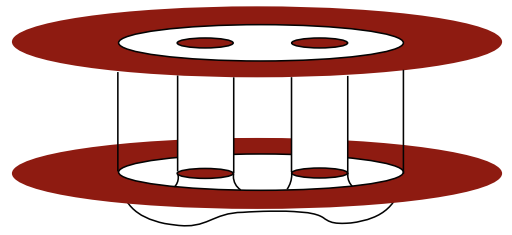

Fig. 15. The analogue of thermal AdS for the pair of pants wormhole. In this case three copies of Lorentzian $\mathrm{AdS}_{3}$ are attached to the three boundaries of the pair of pants

are called the ' $S L(2, \mathbb{Z})$ family' of black holes as they relate to the Euclidean BTZ black hole by a modular transformation. In Appendix B we however show that none of these solutions can be used in the real-time gauge/gravity prescription as the Euclidean part associated with a vertical segment of the QFT contour. The reason is that the matching conditions force the Lorentzian bulk metric to be complex and this results in a energy momentum tensor that does not satisfy the correct reality conditions.

For a higher genus Riemann surface there also exists a similar family of solutions [42] as well as the aforementioned non-handlebody solutions. As for the other fillings of handlebody-type, we expect that only the analogues of the BTZ and the thermal AdS would be relevant for holography of the Lorentzian wormholes. The analogue of thermal AdS is obtained by attaching $m$ copies of empty Lorentzian AdS to the $m$ boundary components of the handlebody. The case of a pair of pants wormhole is sketched in Fig. 15. For the non-handlebodies the corresponding Lorentzian solution remains to be investigated.

6.2. Rotating wormholes. In the previous sections we considered non-rotating wormholes. Rotating wormholes do exist $[43,44]$ and are obtained by taking a quotient with respect to a group generated by elements of the form $\left(\gamma_{1}, \gamma_{2}\right) \in S L(2, \mathbb{R}) \times S L(2, \mathbb{R})$ with $\gamma_{1} \neq \gamma_{2}$. A similar region like $\widehat{\mathrm{AdS}}_{3}$ exists such that the quotient $\widehat{\operatorname{AdS}}_{3} / \Gamma$ is a good spacetime [22,23] and the metric in the outer regions is isometric to the rotating BTZ metric [23]. The corresponding 'Euclidean spaces' for these wormholes, however, are not so straightforward. A prescription for obtaining them has been proposed in [45] and was critically analyzed in [24]. From the holographic perspective, the reality condition of the bulk fields, especially on the Euclidean caps, should be dictated by the standard reality condition of the dual QFT. In the case of the rotating BTZ we have demonstrated in [5] (Sect. 4.5) that the matching conditions result in a complex metric on the Euclidean caps. It is likely that the same would be true here, namely the Euclidean solution that should be glued to the rotating Lorentzian wormhole would be complex.

There are several issues that need to be resolved in order to understand the rotating case. Firstly, it is not straightforward to find in the rotating wormhole the analogue of a $U=0$ slice of the non-rotating wormhole [23]. One approach to this problem is to consider the rotating wormholes as deformations of the non-rotating wormholes. In the Lorentzian case such deformations might be described by a Lorentzian version of the standard quasiconformal mappings [27], one for each $S L(2, \mathbb{R})$ factor. One would then need to extend these deformations to the 'Euclidean' solutions, which, as mentioned above, are likely to be complex solutions that possess a real slice where the Lorentzian solution can be glued. It would be interesting to further develop this direction. 


\section{Outlook}

We have discussed the holographic interpretation of a class of 2+1-dimensional wormhole spacetimes. They are interesting toy models for the analysis of global issues in the real-time gauge/gravity correspondence. We have shown that the asymptotics of the complete solution that includes both the Lorentzian solution and the Euclidean caps completely characterize the geometry including the regions behind the horizons. This came about by a subtle interplay between global issues and the real-time gauge/gravity dictionary. In particular, the real-time gauge/gravity prescription requires gluing a smoothly Euclidean solution to the Lorentzian solution at early and late times. This in turn fixes the apparent freedom for independent Weyl rescaling at different outer components and results in holographic data that contain information about the complete geometry.

We thus find that the Lorentzian CFT correlators encode in a very precise sense the parts of the geometry that lie behind the horizons. This presents a unique opportunity to study and settle classic questions and puzzles in black hole physics. The way the information is given to us, however, (i.e. in terms of CFT correlators) is very different from the way the black holes puzzles are usually formulated (e.g. using bulk local observers) and this presents the main obstacle in directly addressing these issues.

In this respect, one of the most interesting cases to further understand is that of spacetimes with $m=1$ and $g>0$. As discussed earlier, this has only one outer region. The form of the 1-point and 2-point functions indicate entanglement between the outer region and the region behind the horizon. It is not clear however which modes are entangled in the CFT, since unlike the cases with $m>1$ the dual state seems to be defined in only a single copy of the Hilbert space.

We can however suggest some possibilities. Note that all wormholes can be viewed as quotients of a part of BTZ, since the group $\Gamma$ associated with them always contains a subgroup isomorphic to that of BTZ (namely $\mathbb{Z}$ ) and so one can take the quotient first with respect to this group, resulting in BTZ, and then with respect to the rest of the group elements (modulo issues related to the regions one needs to remove to avoid closed timelike curves that need to be investigated). Thus we find a state in the tensor product of two Hilbert spaces (associated with the two boundaries of BTZ) with certain correlations between the two components because of the final quotient. It would be interesting to make this more precise and understand its relation with the apparent entanglement between the outer and inner regions.

As mentioned earlier, there is a reasonable guess for the dual state: this would be the pure state obtained by performing the Euclidean path integral over the Riemann surface $\Sigma$ that is the conformal boundary of the Euclidean 3-manifold that we glue to the Lorentzian spacetime at $t=0$. However, this appears at odds with the presence of a bulk horizon. It would be interesting to clarify this and also check the identification of the state by computing in the CFT the expectation value of the stress energy tensor in this state and see if the results agree with our bulk computation.

One of the main reasons the black hole entropy has been so puzzling is that classically black holes appear to be unique (they have "no-hair") so their phase space is zero dimensional. In a typical quantum system the correspondence principle relates the quantum states to the classical phase space and the entropy of the system to the volume of phase space in Planck units. Thus since the phase space for black holes appears to be zero dimensional, they should not carry any entropy. As was discussed earlier, however, the outer region of the wormholes is isometric to the BTZ black hole. Thus one can view the 'wormhole' spacetimes with a single outer region as 'BTZ hair', where the 'hair' is essentially the non-trivial topology hidden behind the horizon. It is thus natural to ask 
whether this classical phase space can account for the entropy of the BTZ black hole upon quantization. ${ }^{9}$ In other words, these spacetimes would then be the semi-classical approximation of the underlying black hole microstates. This is similar in spirit to the fuzzball proposal (whose relation to holography was discussed extensively in the review [47]) although here the geometries counted contain horizons and singularities.

Let us outline how one would do such a computation. We have seen that these spacetimes are uniquely specified by a Riemann surface with one boundary and the mass of the BTZ black hole is determined by one of the moduli of the Riemann surface. Thus the classical phase space is the moduli space of Riemann surfaces of arbitrary genus with a single fixed modulus, corresponding to the length of the horizon (in other words the BTZ mass parameter), which is the only parameter accessible to an observer outside of the horizon. More precisely, if one uses the Fenchel-Nielsen coordinates on the Teichmüller space (described in detail in Appendix A.1) the restriction to a fixed BTZ mass amounts to considering a codimension one hypersurface in Teichmüller space. This hypersurface is invariant under the mapping class group and therefore directly descends to the moduli space. The complete phase space is then the union of these hypersurfaces for different genera. Classically, the volume of this phase space is infinite and one should proceed by geometric quantization. One can readily compute the symplectic form on the covariant space following [48-50] and proceed to quantize. It would be interesting to carry out this computation. The explicit form of the metric derived in Appendix A should facilitate this.

Acknowledgments. We would like to thank Alex Maloney, Jan Smit and Erik Verlinde for discussions. KS acknowledges support from NWO via a VICI grant.

Open Access This article is distributed under the terms of the Creative Commons Attribution Noncommercial License which permits any noncommercial use, distribution, and reproduction in any medium, provided the original author(s) and source are credited.

\section{A. Coordinate Systems}

The description of the wormholes in Sect. 2 as a quotient $\widehat{\operatorname{AdS}}_{3} / \hat{\Gamma}$ is precise but rather abstract. This Appendix presents a metric description of the wormholes, building on [24]. More details are presented in [51]. Concretely, this description consists of covering the spacetime with a set of charts for which the coordinates have natural ranges. We then show that on each of these charts we can put an explicit metric, which features several natural parameters that describe the local geometry (similar to the mass $M$ for a BTZ metric). We will show that one may arrive at a complete description of the spacetime by combining the parameters from all the charts plus specifying some combinatorial data, which can be combined in a single labelled fatgraph. An example of such a fatgraph is given in Fig. 2, which completely describes a spacetime with the topology sketched in Fig. 1. The particular parameters that will appear in the metric are very similar to Fenchel-Nielsen coordinates on Teichmüller space, so we begin with a review of these coordinates.

A.1. Fenchel-Nielsen coordinates. In this section we review the definition of the Fenchel-Nielsen coordinates on the Teichmüller space of Riemann surfaces of genus $g$ with $m>0$ circular boundaries (and no punctures). As we discussed in the main text,

\footnotetext{
${ }^{9}$ This question has been independently pursued by Alex Maloney [46].
} 


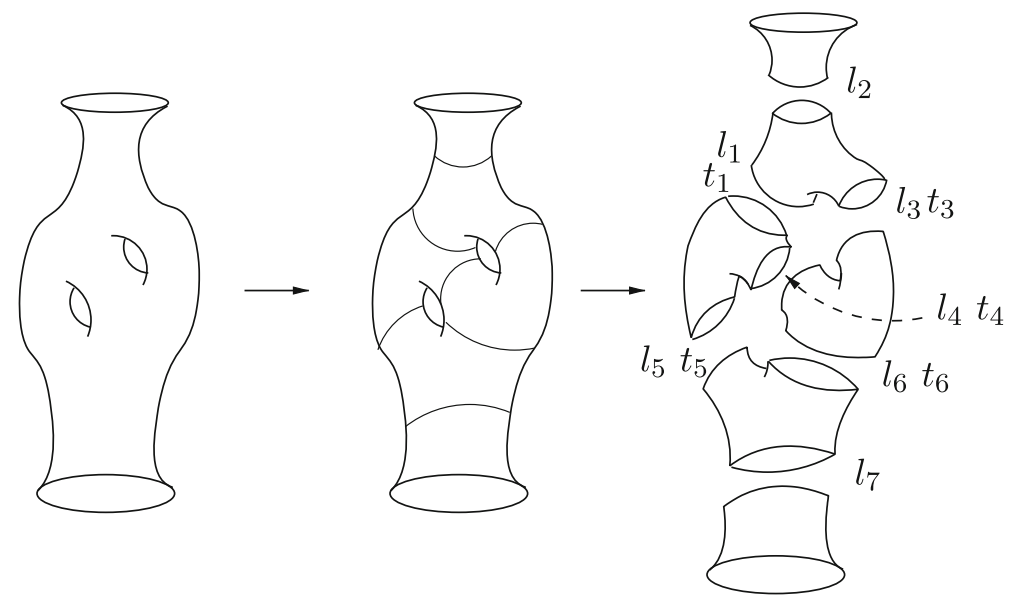

Fig. 16. Defining Fenchel-Nielsen coordinates on a Riemann surface. We cut the Riemann surface into pairs of pants along simple closed geodesics and assign lengths $l_{i}$ to all the edges of every pants plus a twisting parameter $t_{j}$ for every gluing involving two pairs of pants

all such Riemann surfaces are quotients of the upper half plane, from which they all inherit a canonical metric of constant negative curvature.

It can be shown that in this metric there is precisely one smooth periodic geodesic corresponding to every nontrivial primitive loop on the surface. After a little counting one finds that one can pick a maximum of $3 g-3+2 m$ of such periodic geodesics that do not intersect each other, see Fig. 16 for an example. We then $c u t$ the Riemann surface along these geodesics, i.e. we remove these geodesics from the surface. This leaves us with $2 g-2+m$ disconnected so-called 'pairs of pants', that is Riemann surfaces of genus 0 with three circular boundary components, as well as $m$ annuli. The annuli correspond to the regions on the Riemann surface between a periodic geodesic that is retractable into a boundary component and the boundary component itself.

The Fenchel-Nielsen coordinates are now based on the idea that we can reconstruct the complete Riemann surface from this collection of pairs of pants and annuli, provided we also specify how to glue these 'building blocks' together. Therefore, we can define coordinates on the Teichmüller space of Riemann surfaces of the given type by specifying enough data to first of all construct the pairs of pants and annuli that make up the original surface, plus some rules on how to glue them together.

Let us begin with a description of the individual pairs of pants and annuli. Using some simple hyperbolic geometry, see for example [25], one finds that the pairs of pants are completely described by only three real moduli which one may take to be the strictly positive lengths of the periodic geodesics along which we made the cuts. A similar statement is true for the annuli: these are completely specified by giving the length of the periodic geodesic as well. Since we cut along $3 g-3+2 m$ periodic geodesics, we find that we can reconstruct the individual pairs of pants and annuli by the specification of precisely $3 g-3+2 m$ strictly positive lengths.

Next, we have to specify the way in which the various components are glued together. More specifically, we have to specify the angle that the various components have to be twisted with before we perform the gluing. Notice that these angles are actually only relevant when we glue two pairs of pants together, since twisting an annulus is an isometry. The angles are defined as follows, see Fig. 17. On every pair of pants we may define 


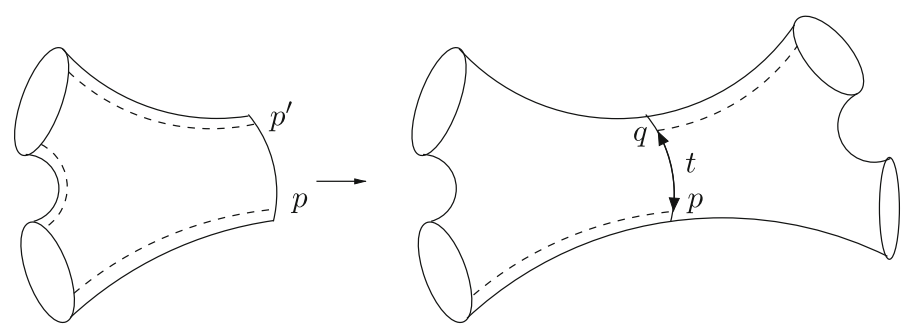

Fig. 17. The twist parameter $t$ is defined by the angle between two points $p$ and $q$ that lie at the intersection of the dashed geodesics with a boundary circle

three distinguished geodesics, namely the shortest non-intersecting geodesics that run from one boundary circle to another. A given boundary circle of the pants intersects with two of these geodesics, say at the points $p$ and $p^{\prime}$. (Figure 17 is drawn slightly distorted since these points actually lie diametrically opposite of each other. This follows from a reflection isometry of the pair of pants whose fixed points are precisely the three geodesics we just defined.) Following the same reasoning on the other pair of pants we find two more points, say $q$ and $q^{\prime}$, on this boundary circle. The twist parameter describing the gluing is now precisely the angle between, say $p$ and $q$, on the boundary circle. ${ }^{10}$

Since we cut along $3 g-3+2 m$ geodesics, we have as many gluings to perform. For precisely $m$ of these we glue annuli to pairs of pants, which leaves us with $3 g-3+m$ gluings between pairs of pants for which we need to specify an angle. Adding these to the $3 g-3+2 m$ lengths precisely gives the required number of $6 g-6+3 m$ parameters. Indeed, it can be shown that these lengths and angles provide good coordinates that cover the Teichmüller space of Riemann surfaces of the given type, which is therefore isomorphic to $\left(\mathbb{R}^{+}\right)^{3 g-3+2 m} \times \mathbb{R}^{3 g-3+m}$. This is then the Fenchel-Nielsen description of the Teichmüller space.

A.2. Construction of the charts. The procedure to obtain our charts is sketched in Fig. 18 and is described in words as follows. We first restrict ourselves to the $U=0$ Riemann surface $S=H / \Gamma$. Just as in the Fenchel-Nielsen description of the surface, we begin by picking a maximal set of $3 g-3+2 m$ primitive periodic geodesics. We now consider one geodesic and 'thicken' it, i.e. we define a small cylindrical neighborhood around the geodesic. When we try to extend this 'collar' further, eventually we might wrap another cycle and the cylinder will then start to overlap with itself. We then stop the thickening when the boundary circles just touch each other, as indicated in Fig. 18. In the cases where the periodic geodesic we consider is retractable into a boundary component we extend the thickening on that end all the way to this boundary. Except for the BTZ black hole, the other end of the cylinder is then never extendable to another boundary component and pinches as usual.

This procedure results in two types of cylindrical domains: those where both boundary circles are pinched on $S$, which we call 'inner domains', and those where precisely one end extends to a boundary component, which we call 'outer domains'. An inner domain covers part of two pairs of pants, whereas an outer domain covers an annulus and part of a pair of pants.

10 A shift of $2 \pi$ in the angles corresponds to an element of the mapping class group and therefore to two different points in Teichmüller space. Strictly speaking, therefore, these angles take values in $\mathbb{R}$ in order to properly parametrize the Teichmüller space. We will be rather loose in this distinction. 


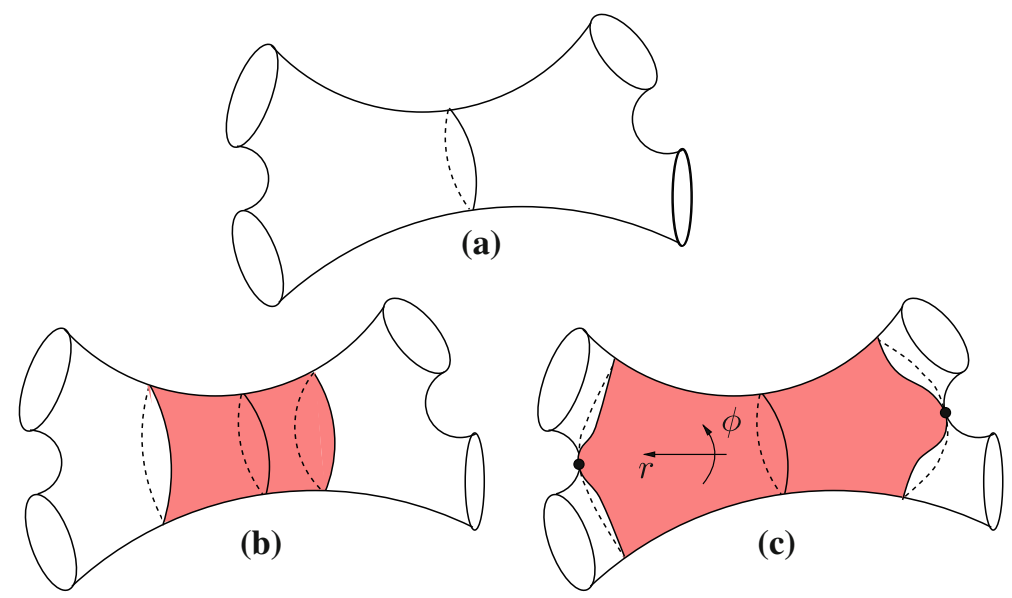

Fig. 18. (a) Charts are defined around a closed periodic geodesic on the Riemann surface. (b) We begin by thickening this geodesic to obtain a cylinder. (c) We extend the cylinder as far as possible, until the bounding circles just touch, in this case on the black dots. We define coordinates $(r, \phi)$ as indicated, as well as a third time coordinate which is not shown

Notice that the inner and outer domains we define here are not precisely the inner and outer regions we defined in the main text. Namely, the inner and outer regions in the main text were separated by the horizons, whereas the outer domains we define here do extend beyond the horizons. The inner domains that we define here never cross the horizons and therefore lie entirely in what we called the inner region in the main text.

Consider now a single pair of pants. It intersects with precisely three (inner or outer) domains, namely those that are defined around each of its boundary circles. Of course, the domains overlap with each other on the pants but more importantly it can be shown that the entire pair of pants is covered by these three domains. (This follows from direct computation using hyperbolic geometry, see [51] for details.) Since the domains also cover the annuli completely, it follows that the entire surface at $U=0$ is covered by these domains. Below, we will use these domains as the $U=0$ slice of analogously defined three-dimensional coordinate patches, which taken together cover the entire spacetime. We will then find a suitable coordinate system on these patches to complete our description of the wormholes.

To define more precisely the inner and outer domains let us lift them to the universal cover $H$ of $S$, where we will use a complex coordinate $z$. Consider one of the periodic geodesics around which we defined a chart. We assume that on $H$ the homotopy class of the periodic geodesic is generated by the identification

$$
\gamma: z \mapsto \lambda z
$$

which can always be realized using the conjugation freedom of $\Gamma$. If $z=x+i y$, then the periodic geodesic lifts to the line $x=0$. The corresponding lift of the cylindrical neighborhood around it is a region $D$ given by

$$
D:-\beta y<x<\alpha y,
$$

for some positive real $\alpha$ and $\beta$ (which are given in terms of the Fenchel-Nielsen parameters that fix the geometry of the pairs of pants, as it will become clear from the analysis below). For an inner domain $\alpha$ and $\beta$ are finite whereas for an outer domain either $\alpha$ or 


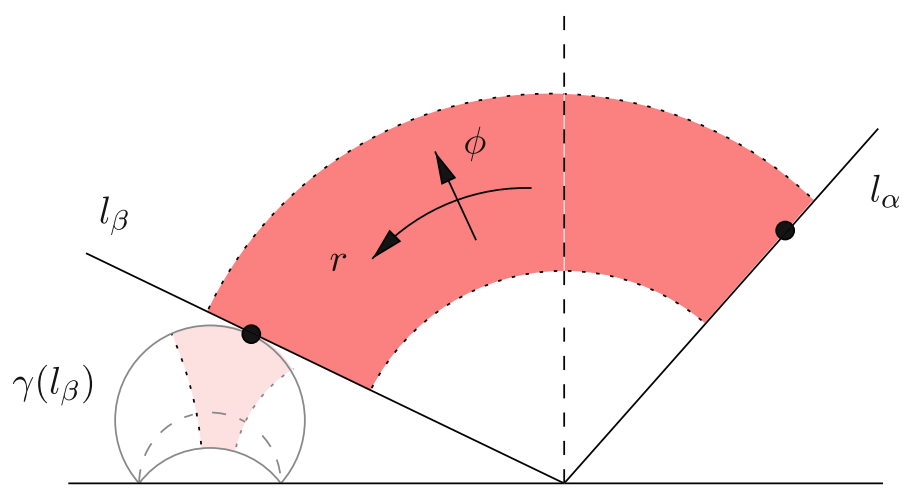

Fig. 19. A lift to the upper half plane of the cylindrical region. The dotted lines bounding the darker region should be identified under the map $w \sim \lambda w$. Under $\gamma_{\beta}$, the region maps to the smaller, lighter shaded region on the left. The image $\gamma\left(l_{\beta}\right)$ of $l_{\beta}$ just touches $l_{\beta}$ at the indicated point. A similar thing happens on the right for $l_{\alpha}$, but we have not sketched the second image

$\beta$ are equal to $+\infty$ and the domain extends all the way to the boundary. A region $D$ with finite $\alpha$ and $\beta$, i.e. corresponding to an inner domain, is sketched in Fig. 19. The lines $l_{\alpha}$ and $l_{\beta}$, given by $\alpha y=x$ and $-\beta y=x$ respectively, determine the bounding circles of the cylindrical neighborhood. We have deliberately chosen the shape of these bounding circles such that they lift to straight lines on $H$ which are called hypercycles. (Recall that geodesics on $H$ are either semicircles that are orthogonal to the real axis or straight vertical lines; hypercycles, on the other hand, are straight lines or circle segments that end on the real axis but not at a right angle. Examples are $l_{\alpha}, l_{\beta}$ and $\gamma\left(l_{\beta}\right)$ in Fig. 19.) As we mentioned above, the cylindrical neighborhood is 'maximally extended' in the sense that its bounding circles on $S$ touch themselves somewhere on $S$. Correspondingly, there must exist $\gamma_{\alpha}, \gamma_{\beta} \in \Gamma$ that map $l_{\alpha}, l_{\beta}$ to circle segments that just touch $l_{\alpha}, l_{\beta}$ on $H$. We have sketched this in Fig. 19.

The cylinder can now be extended to a region on the full three-dimensional wormhole geometry. We first extend the action of the isometry (75) to the Poincaré patch:

$$
\hat{\gamma}:(t, x, y) \sim \lambda(t, x, y),
$$

and then extend the domain $D$ to an invariant domain $\hat{D}$ in the full three-dimensional geometry. For inner domains it is defined as

$$
\hat{D}:-\beta \sqrt{y^{2}-t^{2}}<x<\alpha \sqrt{y^{2}-t^{2}}
$$

with $y^{2}-t^{2}>0$. For outer domains either $\alpha$ or $\beta$ are equal to $+\infty$ and correspondingly there is no restriction on the sign of $y^{2}-t^{2}$ when $x>0$ or $x<0$, respectively. On that end the outer domain extends all the way to the conformal boundary of the spacetime.

We note that the region with $-t^{2}+x^{2}+y^{2} \leq 0$ has to be excluded because it lies within the future and past lightcone of the origin, which is a fixed point of the isometry (77). One may check that (77) indeed leads to closed timelike or lightlike curves in this region. There are other excluded regions that are bounded by lightcones with their vertex at the point at infinity but these are precisely the regions in $\mathrm{AdS}_{3}$ that are not covered by the Poincaré coordinate system anyway. 
Let us now sketch a proof for the covering of the entire spacetime by these domains. First of all, notice that the future and past Cauchy development of the $t=0$ slice (which we will call $C$ ) is covered by the part of the Poincaré coordinate system with $y^{2}-t^{2}>0$. Then from (78) we see that the inner charts all lie within this domain. The domain $C$ can be foliated with slices of constant $U=t / y$ on which the quotient group $\hat{\Gamma}$ acts just as on the initial $U=0$ surface. The covering of $C$ then follows straightforwardly from the fact that the $U=0$ surface is covered.

However, the wormholes are not globally hyperbolic and a part of the wormhole spacetime near the conformal boundary lies outside of $C$. To find the shape of this part of the spacetime we notice the following. Near the conformal boundary the spacetime has the form of an annulus times a time coordinate and when we move inward this annulus pinches just as in Fig. 18c. It follows from (78) that the pinching occurs either at $x=-\beta \sqrt{y^{2}-t^{2}}$ or at $x=\alpha \sqrt{y^{2}-t^{2}}$ for some finite $\alpha, \beta$. Either way this 'pinching surface' must lift to a region with $y>|t|$ and therefore always lies entirely within $C$. It follows that the parts of the spacetime outside of $C$ must have the shape of an annulus times time. It is then easy to verify that these regions of the spacetime outside of $C$ can be described in Poincaré coordinates by starting with the region where $y<|t|$ and $x>0$, excluding the lightcones where $-t^{2}+x^{2}+y^{2} \leq 0$ and taking the quotient of the remainder with respect to the cyclic group generated by (77). Indeed, the domains so obtained are bounded by the lightlike surfaces $y=|t|$ that bound $C$, extend all the way to the conformal boundary $y=0$ and the action of the cyclic covering group guarantees that the quotient has the form of an annulus times time. These regions are by construction also completely covered by an outer domain and therefore indeed the entire spacetime is covered.

One may also explicitly verify that the coordinate systems on the inner and outer domains as given in (79) and (87) below are everywhere well-defined on these domains.

A.2.1. Coordinate systems on inner domains. We may now define new coordinates on the three-dimensional domains $\hat{D}$. For inner domains we define a coordinate system $(\tau, r, \phi)$ via:

$$
\tanh (\tau)=\frac{t}{y}, \quad \mu r+v=\frac{x}{\sqrt{y^{2}-t^{2}}}, \quad e^{2 \sqrt{M} \phi}=-t^{2}+x^{2}+y^{2},
$$

with coefficients

$$
e^{2 \pi \sqrt{M}}=\lambda, \quad \mu+v=\alpha, \quad \mu-v=\beta
$$

From (77) and (78) we find the coordinate ranges:

$$
\tau \in \mathbb{R}, \quad \phi \sim \phi+2 \pi, \quad r \in[-1,1],
$$

and the metric takes the form:

$$
d s^{2}=\frac{1}{\cosh ^{2}(t)}\left(-d t^{2}+\frac{\mu^{2} d r^{2}}{(\mu r+v)^{2}+1}+M\left(1+(\mu r+v)^{2}\right) d \phi^{2}\right) .
$$

This metric already features several parameters $M, \mu, v$ which inform us about the geometry at least in this local patch. We can however introduce one more parameter which is related to the Fenchel-Nielsen twist described above. 


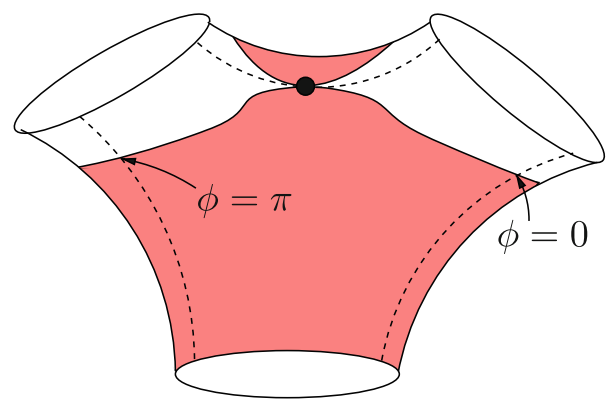

Fig. 20. The phase in $\phi$ can be fixed by letting $\phi=0$ correspond to the intersection of the boundary of the chart with the unique shortest length geodesic (dashed line) between the two boundary circles of the corresponding pair of pants

To find this parameter, let us begin by considering one edge of a particular chart, say at $r=+1$. As indicated in Fig. 20, such an end lies at a pair of pants that is used in the Fenchel-Nielsen description of the surface. We described before that there are three shortest geodesics on this pair of pants that run between the three boundary components, see Fig. 17. As we sketched in Fig. 20, two of these geodesics intersect the boundary circle of the chart. We can now shift $\phi$ such that one of these intersection points corresponds to $\phi=0$ and from the aforementioned reflection symmetry it follows that the other one automatically lies at $\phi=\pi$. (It can also be shown that the third of these geodesics precisely touches the boundary of the charts at the pinching point which is indicated by the black dot in Fig. 20.)

After having implemented this shift at the side $r=+1$ we find that the corresponding points at the side $r=-1$, which lie on another pair of pants, generally lie at a value $\phi=\phi_{0}$ and $\phi=\phi_{0}+\pi$, all modulo $2 \pi$. In fact, the angle $\phi_{0}$ is precisely the FenchelNielsen twist coordinate (denoted $t$ above) that is associated to the gluing. We can make this twist explicit in the metric by introducing a new 'twisted' coordinate $\psi$ given by:

$$
\exp (\sqrt{M} \psi-k)=\exp (\sqrt{M} \phi) f(\mu r+v, \chi)
$$

with

$$
f(\rho, \chi)=\frac{\rho \sin (\chi)+\sqrt{\rho^{2}+\cos ^{2}(\chi)}}{\sqrt{\rho^{2}+1}} .
$$

This coordinate transformation features two new parameters $k$ and $\chi$. If they are chosen such that

$$
e^{-k}=f(\mu+\nu, \chi)=e^{\sqrt{M} \phi_{0}} f(-\mu+\nu, \chi),
$$

then the aforementioned distinguished points are given by $\psi=0$ and $\psi=\pi$ on both sides. The coordinate range of $\psi$ is the same as $\phi$, so $\psi \sim \psi+2 \pi$. The parameter $\chi$ now shows up explicitly in the metric, which takes the form:

$$
\begin{gathered}
d s^{2}=\frac{1}{\cosh ^{2}(t)}\left(-d t^{2}+\frac{\mu^{2} d r^{2}}{(\mu r+v)^{2}+\cos ^{2}(\chi)}+M\left(1+(\mu r+v)^{2}\right) d \psi^{2}\right. \\
\left.-\frac{2 \mu \sqrt{M} \sin (\chi)}{\sqrt{(\mu r+v)^{2}+\cos ^{2}(\chi)}} d \psi d r\right) .
\end{gathered}
$$


This is the final metric on the inner chart. The four different parameters $M, \mu, v, \chi$ appearing in it inform us about some 'local' geometrical aspects of the spacetime. Namely, the periodic geodesic around which we defined the chart lies at the point $r=-v / \mu$ and has length $2 \pi \sqrt{M}$. The angle $\chi$ reflects the twisting of the pairs of pants with respect to each other and the parameters $\mu$ and $v$ are related to the shapes of these pairs of pants: for example, the distance between the periodic geodesic and the pinched hypercycle at $r=1$ is

$$
\left|\ln \left(\frac{\mu+v+\sqrt{(\mu+v)^{2}+1}}{v+\sqrt{v^{2}+1}}\right)\right|,
$$

and the distance to the hypercycle at $r=-1$ has the same form with the replacement $\mu \rightarrow-\mu$.

A.2.2. Coordinate systems on outer domains. For the outer domains we may always conjugate $\Gamma$ such that $\alpha=\infty$ and $\beta$ is finite. We can then use a $(\tilde{\tau}, \rho, \varphi)$ coordinate system defined as

$$
\tanh (\sqrt{M} \tilde{\tau})=\frac{t}{\sqrt{y^{2}+x^{2}}}, \quad \rho=\sqrt{M} \frac{x}{y}, \quad e^{2 \sqrt{M}(\varphi-h)}=-t^{2}+x^{2}+y^{2},
$$

where, as in (80),

$$
e^{2 \pi \sqrt{M}}=\lambda
$$

which is again related to the length of the periodic geodesic. The parameter $h$ shifts the coordinate $\varphi$ such that the aforementioned special points on the bounding circle lie again at $\varphi=0$ and $\varphi=\pi$. We will not need the explicit value of $h$ below. The bounding circle itself is given by

$$
\frac{\cosh (\sqrt{M} \tilde{\tau}) \rho}{\sqrt{\rho^{2}+M}}=-\frac{\beta^{2}}{1+\beta^{2}},
$$

and the coordinate ranges are given by

$$
\tilde{\tau} \in \mathbb{R}, \quad \varphi \sim \varphi+2 \pi, \quad \frac{\cosh (\sqrt{M} \tilde{\tau}) \rho}{\sqrt{\rho^{2}+M}}>-\frac{\beta^{2}}{1+\beta^{2}} .
$$

The radial boundary of the spacetime lies at $\rho \rightarrow \infty$. The metric takes the form:

$$
d s^{2}=\frac{\rho^{2}+M}{\cosh ^{2}(\sqrt{M} \tilde{\tau})}\left(-d \tilde{\tau}^{2}+d \varphi^{2}\right)+\frac{d \rho^{2}}{\rho^{2}+M} .
$$

Notice that these coordinate systems extend beyond the future and past horizons, which lie at the surfaces $x=|t|$ or

$$
\rho=\sqrt{M}|\sinh (\sqrt{M} \tilde{\tau})| .
$$

The metric in the region outside of these horizons (which we called the outer region in the main text) can be put back in BTZ form (20) by the coordinate transformation:

$$
r^{2}=\frac{\rho^{2}+M}{\cosh ^{2}(\sqrt{M} \tilde{\tau})}, \quad \tanh (\sqrt{M} t)=\sqrt{1+M / \rho^{2}} \tanh (\sqrt{M} \tilde{\tau}), \quad \phi=\varphi .
$$

Notice that the parameter $M$ in (91) agrees with the BTZ mass $M$. 
A.3. Parameters. The above charts can be combined to cover the wormhole spacetime completely. More specifically, for a wormhole of genus $g$ and with $m$ boundaries, we can cover the entire spacetime with $3 g-3+m$ inner charts plus $m$ outer charts. For every inner chart we have four parameters, $M, \mu, v, \chi$, and for every outer chart we have a single parameter $M$. As we showed above, the angles $\chi$ and the parameters $M$ are directly related to the Fenchel-Nielsen twists and length parameters associated to the periodic geodesics and should therefore completely determine the surface. The remaining $\mu$ and $v$ parameters are therefore expressable in terms of those.

The precise relation takes the following form. Consider a pair of pants in the surface. In our description of the surface it is covered by three (inner or outer) charts, in fact it is already completely covered by only half of each of these three charts. Suppose now that chart number 3 is an inner chart (with parameters $\mu_{3}, \nu_{3}, M_{3}, \chi_{3}$ ) and that it is the half with $r>0$ that lies on the pair of pants under consideration. Denote the $M$ parameters in the other two charts as $M_{i}$ with $i \in\{1,2\}$. One then finds the relation:

$$
\mu_{3}+\nu_{3}=\frac{\sqrt{C_{1}^{2}+C_{2}^{2}+2 C_{1} C_{2} C_{3}}}{\sinh \left(\pi \sqrt{M_{3}}\right)},
$$

with $C_{i}=\cosh \left(\pi \sqrt{M}_{i}\right)$. This relation follows from a straightforward computation in the upper half plane using hyperbolic geometry. A similar relation can be found at the other side of chart number 3, which has $r<0$ and lies on another pair of pants. Namely, using the parameters $M_{1}^{\prime}$ and $M_{2}^{\prime}$ of the two other charts on that pair of pants we find:

$$
-\mu_{3}+\nu_{3}=\frac{\sqrt{C_{1}^{\prime 2}+C_{2}^{\prime 2}+2 C_{1}^{\prime} C_{2}^{\prime} C_{3}}}{\sinh \left(\pi \sqrt{M_{3}}\right)},
$$

with $C_{i}^{\prime}=\cosh \left(\pi \sqrt{M_{i}^{\prime}}\right)$. Using these formulae, we can determine all the $\mu, v$ parameters in the inner charts if we are only given the $M$ parameters in every chart. This reduces the number of independent parameters to two per inner chart and still one per outer chart, just as for the Fenchel-Nielsen description of the surface.

A.4. Fatgraph description. To completely specify the spacetime we need to specify both the parameters and the way the charts are glued together. This combinatorial data can be nicely summarized in an oriented trivalent fatgraph as shown in Fig. 2. (In the usual Fenchel-Nielsen description of the surface this combinatorial data is implicitly specified, for example by using a reference surface. The description given below, on the other hand, explicitly fixes the required combinatorial data and it is then no longer necessary to use a reference surface.)

The data in the fatgraph is translated to the coordinate systems as follows. Every edge represents a periodic geodesic and therefore a chart. Every vertex represents a pair of pants. The orientation of the edges indicates the direction of increasing $r$ (and by convention always points outward for outer charts), and the 'fattening' is necessary to indicate how three charts come together on a pair of pants. If we add to this fatgraph two parameters $M, \chi$ for every interior edge of the graph and a single parameter $M$ for every outer edge, then the wormhole spacetime is completely specified.

At this point we should note that there are two discrete ambiguities in the above definitions of the coordinates $\psi$ and $\varphi$ on the inner and outer charts that we have not yet 


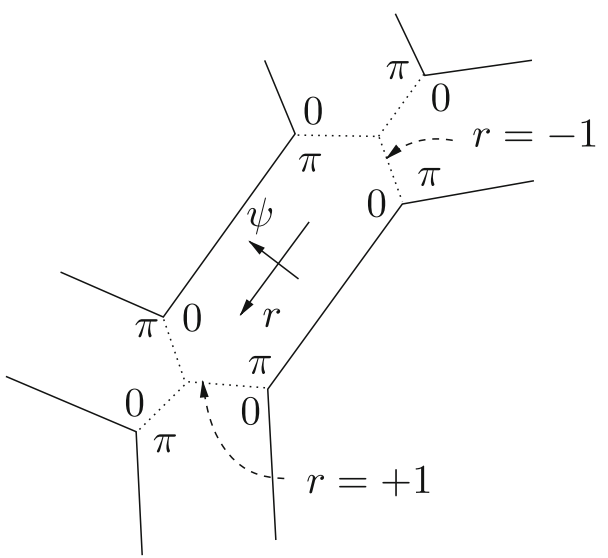

Fig. 21. Fixing the ambiguities in the definition of $\psi$ and $\varphi$

dealt with. Although these ambiguities do not affect the metric or the coordinate ranges given above, they will affect the transition functions below and therefore they should be fixed.

The first ambiguity involves the direction of increasing $\psi$ and $\varphi$. With the fatgraph description this can be easily fixed by fixing the handedness of the $(r, \psi)$ or $(r, \varphi)$ coordinate system to be the same in every chart.

The second ambiguity is the fact that we have only $\psi$ or $\varphi$ up to an overall shift by $\pi$. To see this, recall that we decided that the point $\psi=0$ or $\varphi=0$ would correspond to one of the distinguished points on the boundary circle (sketched in Fig. 20) and by the reflection isometry the other point would then be at $\psi=\pi$ or $\varphi=\pi$. We however did not yet specify which point we chose to be at 0 and which one at $\pi$. This ambiguity can be fixed from the fatgraph. We first demand that at an overlap between two charts the point where $\psi=0$ on one chart corresponds to $\psi=\pi$ on the other chart (and similarly for $\varphi$ ), as indicated in Fig. 21. Furthermore, for an inner chart we should alternately associate $\psi=0$ and $\psi=\pi$ to the four corners of the corresponding edge in the fatgraph, which is indicated in Fig. 21 as well. This fixes the ambiguity up to an overall shift of $\psi$ or $\varphi$ with $\pi$ in all charts at the same time, which is however irrelevant for the description of the manifold.

A.5. Transition functions. With all the ambiguities fixed, we may proceed to define transition functions on the overlap between two different charts. These follow from the coordinate transformations (79) and (87) plus the explicit form of the elements of $\hat{\Gamma}$ in Poincaré coordinates (which can be deduced from (15) and (16).

An important subtlety is that we find different transition functions depending on the gluings and the orientations of the charts. For example, if we consider the vertex in Fig. 2 where we may go from chart 2 to chart 3 or chart 4, we find different transition functions because we turn 'right' at the vertex if we go to chart 3 , whereas we turn 'left' if we go to chart 4. As another example, the transition functions between chart 2 and chart 3 (on both vertices) are different from those between chart 5 and chart 6 because (again on both vertices) the orientation of chart 3 and chart 6 are not the same. When we define the transition functions below we will have to take into account these different possibilities. 

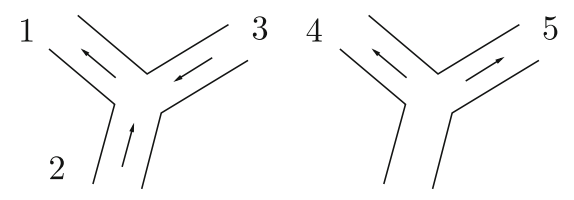

\begin{tabular}{ccccc} 
& $\epsilon$ & $\epsilon^{\prime}$ & $d$ & $d^{\prime}$ \\
\hline $1 \rightarrow 2$ & -1 & +1 & 0 & $\pi$ \\
$1 \rightarrow 3$ & -1 & +1 & $\pi$ & 0 \\
$2 \rightarrow 1$ & +1 & -1 & $\pi$ & 0 \\
$2 \rightarrow 3$ & +1 & +1 & 0 & $\pi$ \\
$3 \rightarrow 1$ & +1 & -1 & 0 & $\pi$ \\
$3 \rightarrow 2$ & +1 & +1 & $\pi$ & 0 \\
$4 \rightarrow 5$ & -1 & -1 & $\pi$ & 0 \\
$5 \rightarrow 4$ & -1 & -1 & 0 & $\pi$
\end{tabular}

Fig. 22. Possible transitions between inner charts. The transition functions are by definition always taken from unprimed to primed coordinate systems: for example, in the first line the unprimed coordinates in (96) are the coordinates in chart 1 and the primed coordinates are those of chart 2

In the transition functions we will not use the 'twisted' coordinate $\psi$ defined in (83). Instead, we will use the coordinate $\phi$ which agrees with $\psi$ at the bounding circle of the chart where we define the transition function. Of course, it is not hard to compose the transition functions with (83) and its inverse, or a similar function when the transition takes place at $r=-1$.

A.5.1. Transitions between two inner charts. The complete set of possibilities for the transitions between two inner charts is depicted in Fig. 22. As one may expect, the transition functions are almost the same for either one of these possibilities and it is convenient to give them in a general form with certain parameters $\epsilon, \epsilon^{\prime}, d$ and $d^{\prime}$ whose value depends on these possibilities and is given in the table in Fig. 22. Using these parameters, one finds for the transition functions,

$$
\begin{aligned}
t^{\prime} & =t \\
-\epsilon^{\prime}\left(\mu^{\prime} r^{\prime}+v^{\prime}\right) & =\cosh (A) \epsilon(\mu r+v)-\sinh (A) \sqrt{(\mu r+v)^{2}+1} \cosh (\epsilon \sqrt{M}(\phi-d)) \\
e^{2 \epsilon^{\prime} \sqrt{M^{\prime}}\left(\phi^{\prime}-d^{\prime}\right)} & =\frac{\epsilon(\mu r+v)-\sqrt{(\mu r+v)^{2}+1} \cosh (\epsilon \sqrt{M}(\phi-d)-g)}{\epsilon(\mu r+v)-\sqrt{(\mu r+v)^{2}+1} \cosh (\epsilon \sqrt{M}(\phi-d)+g)}
\end{aligned}
$$

with

$$
\cosh (A)=\frac{\cosh (\pi \sqrt{M}) \cosh \left(\pi \sqrt{M^{\prime}}\right)+\cosh \left(\pi \sqrt{M^{\prime \prime}}\right)}{\sinh (\pi \sqrt{M}) \sinh \left(\pi \sqrt{M^{\prime}}\right)}
$$

and

$$
\sinh (A) \sinh (g)=1 \text {. }
$$

Here $M$ and $M^{\prime}$ denote mass parameters in the metric on the unprimed and the primed chart between which we define the transition functions, and $M^{\prime \prime}$ denotes the mass parameter from the metric of the third chart that joins this vertex. We therefore have to inspect the metric of all three charts at the vertex in order to obtain the transition functions between only two of these charts. 


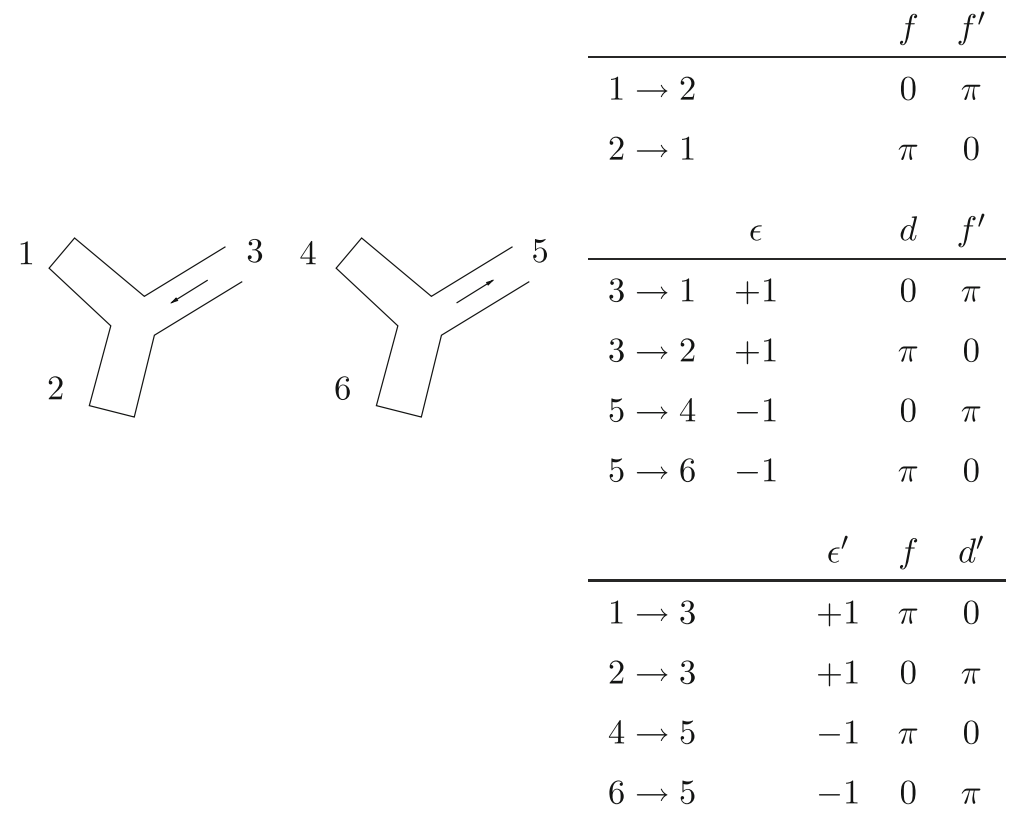

Fig. 23. Possible transitions involving outer charts. In this picture the charts $1,2,4$ and 6 are outer charts and the charts 3 and 5 are inner charts. Conventions are as in Fig. 22

Notice that the transition functions are not automatically periodic in $\phi$ or $\phi^{\prime}$; they are in fact only valid for $\phi, \phi^{\prime} \in[0,2 \pi)$. Of course, this is by no means a restriction as this is sufficient to cover the entire chart. The other boundaries of the domain of validity of the transition functions are obtained from the coordinate ranges (81). For example, substituting $r^{\prime}=1$ in the second equation of (96) one finds an equality involving $r$ and $\phi$ which defines the boundary of the domain of definition of the transition functions.

A.5.2. Transitions involving outer charts. If the transitions involve outer charts we need the $(\tilde{\tau}, \rho, \varphi)$ coordinate system. Since we always pick the $\rho$ coordinate to increase towards the boundary there is no ambiguity on the orientation of this coordinate. We are however still left with the left/right ambiguity and correspondingly need a discrete parameter $f$ associated to every outer chart. For the transition functions between two outer charts we find,

$$
\begin{aligned}
& \rho^{\prime}=-\sqrt{\frac{M^{\prime}}{M}}\left(\cosh (A) \rho+\sinh (A) \cosh (\sqrt{M}(\varphi-f)) \frac{\sqrt{\rho^{2}+M}}{\cosh (\sqrt{M} \tilde{\tau})}\right), \\
& \sqrt{M} \tanh \left(\sqrt{M^{\prime}} \tilde{\tau}^{\prime}\right) \sqrt{\rho^{\prime 2}+M^{\prime}}=\sqrt{M^{\prime}} \tanh (\sqrt{M} \tilde{\tau}) \sqrt{\rho^{2}+M} \\
& e^{2 \sqrt{M^{\prime}}\left(\varphi^{\prime}-f^{\prime}\right)}=\frac{\rho \cosh (\sqrt{M} \tilde{\tau})+\sqrt{\rho^{2}+M} \cosh (\sqrt{M}(\varphi-f)-g)}{\rho \cosh (\sqrt{M} \tilde{\tau})+\sqrt{\rho^{2}+M} \cosh (\sqrt{M}(\varphi-f)+g)},
\end{aligned}
$$

with the possible values of $f$ and $f^{\prime}$ given in Fig. 23 and the same values of $A$ and $g$ as before. The transition function on the second line is slightly implicit but it is straightforward to plug in the solution for $\rho^{\prime}$ of the first line and then solve for $\tilde{\tau}^{\prime}$. 
Similarly, between an inner and an outer chart we find,

$$
\begin{aligned}
& \rho^{\prime}=\frac{\sqrt{M^{\prime}}}{\cosh (t)}\left(\cosh (A) \epsilon(\mu r+v)-\sinh (A) \sqrt{(\mu r+v)^{2}+1} \cosh (\epsilon \sqrt{M}(\phi-d))\right), \\
& \tanh \left(\sqrt{M^{\prime}} \tilde{\tau}^{\prime}\right) \sqrt{\rho^{\prime 2}+M^{\prime}}=\sqrt{M^{\prime}} \tanh (t), \\
& e^{2 \sqrt{M^{\prime}}\left(\varphi^{\prime}-f^{\prime}\right)}=\frac{\epsilon(\mu r+v)-\sqrt{(\mu r+v)^{2}+1} \cosh (\epsilon \sqrt{M}(\phi-d)+g)}{\epsilon(\mu r+v)-\sqrt{(\mu r+v)^{2}+1} \cosh (\epsilon \sqrt{M}(\phi-d)-g)},
\end{aligned}
$$

and conversely,

$$
\begin{aligned}
\sqrt{M} \tanh \left(t^{\prime}\right) & =\tanh (\sqrt{M} \tilde{\tau}) \sqrt{\rho^{2}+M}, \\
\frac{\epsilon^{\prime}\left(\mu^{\prime} r^{\prime}+v^{\prime}\right)}{\cosh \left(t^{\prime}\right)} & =\sqrt{\frac{1}{M}}\left(\cosh (A) \rho+\sinh (A) \cosh (\sqrt{M}(\varphi-f)) \frac{\sqrt{\rho^{2}+M}}{\cosh (\sqrt{M} \tilde{\tau})}\right), \\
e^{2 \epsilon^{\prime} \sqrt{M^{\prime}}\left(\phi^{\prime}-d^{\prime}\right)} & =\frac{\rho \cosh (\sqrt{M} \tilde{\tau})+\sqrt{\rho^{2}+M} \cosh (\sqrt{M}(\varphi-f)+g)}{\rho \cosh (\sqrt{M} \tilde{\tau})+\sqrt{\rho^{2}+M} \cosh (\sqrt{M}(\varphi-f)-g)} .
\end{aligned}
$$

Again, these transition functions are not obviously periodic in $\phi$ and $\varphi$ are are only valid in the interval $[0,2 \pi)$ and the other boundaries are again found by inserting the coordinate ranges (81) and (90) in the transition functions. One may again compose the transition functions with (83) and its inverse to obtain the transition functions for the twisted coordinate $\psi$ on the inner charts.

\section{B. Eternal Black Holes and Filled Tori}

In this Appendix we discuss the genus 1 handlebodies. We show that the ' $S L(2, \mathbb{Z})$ family' of black holes cannot be used in the real-time gauge/gravity prescription as the bulk filling of a vertical segment of the QFT contour because the matching conditions lead to a complex Lorentzian metric (and therefore $\left\langle T_{i j}\right\rangle$ does not satisfy the correct reality conditions, either).

Consider a Euclidean field theory on a torus with modular parameter $\tau=\tau_{1}+i \tau_{2}$. Without loss of generality we can pick the circle given by $z \sim z+1$ as the spatial circle along which we will cut open the Euclidean path integral and glue the Lorentzian solutions. More precisely, we will glue two Lorentzian cylinders to the lines $y=0$ and $y=\tau_{2} / 2$, where $z=x+i y$. As we discussed in [5], $\tau_{1}$ is then $i$ times the angular momentum chemical potential, but since we are not interested in rotating black holes here, we, will set $\tau_{1}$ to zero throughout this Appendix (it is straightforward to generalize to $\tau_{1} \neq 0$ ), so $\tau=i \tau_{2}$ is purely imaginary.

The torus so defined admits multiple bulk fillings, which are given by the specification of a contractible cycle $z \sim z+a \tau+b$ with $(a, b)$ two relatively prime integers. For each of these fillings, one may obtain a complete Euclidean metric which is locally $H^{3}$. After cutting the torus in half, we will glue a Lorentzian bulk solution to the bulk hypersurface ending on the lines $y=0$ and $y=\tau_{2} / 2$. This hypersurface has the shape of an annulus, except when $(a, b)=(0,1)$, when it consists of two disks. In this case the matching Lorentzian solution is two segments of thermal AdS. Notice also that for $(a, b)=(1,0)$ we obtain the rotating BTZ black hole. To find the matching Lorentzian solutions in the general case, we will first explicitly write down the Euclidean bulk metric. We then investigate how the Lorentzian metric is determined by the matching conditions. 
Euclidean geometries. Let us give a brief review of the possible fillings of the torus. We will again use the Poincaré coordinates $(\tau, x, y)$ defined in $(26)$ on $H^{3}$, as well as the complex coordinate $w=x+i \tau$ on the boundary of $H^{3}$. (Notice that the $\tau$ here is a coordinate and not the modular parameter of the torus. We henceforth exclusively use the coordinate $w$ so no confusion should arise.) Any torus handlebody can be obtained as a quotient of $H^{3}$ by a cyclic group of identifications generated in Poincaré coordinates by:

$$
(w, y) \sim\left(e^{2 \pi i \beta} w,\left|e^{2 \pi i \beta}\right| y\right)
$$

with $\beta=\beta_{1}+i \beta_{2}$ a complex number.

Let us now compute the bulk metric when we use the complex boundary coordinate $z$ which has the natural periodicity $z \sim z+1 \sim z+\tau$. We can do so using the map $J$ of Sect. 4. In this case, $J$ is a locally biholomorphic map from $\mathbb{C}$ rather than $H$, since the universal covering of the torus is $\mathbb{C}$ and not $H$. If the contractible cycle is given by $(a, b)$, the corresponding map $J: \mathbb{C} \rightarrow S^{2}$ is given by:

$$
J: z \mapsto w=e^{\alpha z},
$$

with $\alpha=2 \pi i(a \tau+b)^{-1}$. The identifications $z \sim z+1 \sim z+\tau$ become

$$
w \sim w e^{\alpha} \sim w e^{\alpha \tau}
$$

which implies

$$
w \sim e^{\alpha(c \tau+d)} w
$$

Now, since one trivially has that $w \sim e^{\alpha(a \tau+b)} w$, it follows that the single identification (104) is equivalent to both identifications in (103) provided $a d-b c=1$. Comparing (104) with (101), we then read off that

$$
\beta=\frac{c \tau+d}{a \tau+b}
$$

Following the same steps as in Sect. 4, we find that the bulk metric in the $z$ coordinate becomes

$$
d s^{2}=\frac{d \rho^{2}}{\rho^{2}}+\frac{1}{\rho^{2}}\left|d z+\frac{\bar{\alpha}^{2} \rho^{2}}{4} d \bar{z}\right|^{2} .
$$

This metric is of the Fefferman-Graham form (33) and we can read off that the one-point function of the stress energy tensor is given by:

$$
\left\langle T_{z z}\right\rangle=\frac{\alpha^{2}}{2},
$$

which is again $-S[J]$, just as we found for the higher genus handlebodies in Sect. 4. 
Lorentzian geometry. Let us now consider the continuation to the Lorentzian geometry. On the boundary we cut open the Euclidean geometry along the circles given by $y=0$ and $y=\tau_{2} / 2$. In every case except thermal AdS these circles are the boundary of a single annular region in the bulk manifold. Locally the unique solution is simply given by analytic continuation. Using the boundary lightcone coordinates $(u, v)$, we find the Lorentzian metric,

$$
d s^{2}=\frac{d \rho^{2}}{\rho^{2}}+\frac{1}{\rho^{2}}\left(d u+\frac{\bar{\alpha}^{2}}{4} \rho^{2} d v\right)\left(d v+\frac{\alpha^{2}}{4} \rho^{2} d u\right) .
$$

The periodicity for the boundary coordinates is $(u, v) \sim(u+1, v+1)$, and $(u, v)$ are real, whereas $\rho$ has the same range as above. This metric is however complex unless $\alpha^{2}$ is real, which only happens if either $a=0$ or $b=0$. This is problematic both from the bulk and the holographic perspective. In particular, the expectation value of the dual stress energy tensor can be computed using (34),

$$
\left\langle T_{u u}\right\rangle=\frac{1}{2} \alpha^{2}, \quad\left\langle T_{v v}\right\rangle=\frac{1}{2} \bar{\alpha}^{2},
$$

and is complex, which cannot be the case for a hermitian operator.

\section{References}

1. Maldacena, J.M.: The large N limit of superconformal field theories and supergravity. Adv. Theor. Math. Phys. 2, 231-252 (1998)

2. Gubser, S.S., Klebanov, I.R., Polyakov, A.M.: Gauge theory correlators from non-critical string theory. Phys. Lett. B 428, 105-114 (1998)

3. Witten, E.: Anti-de Sitter space and holography. Adv. Theor. Math. Phys. 2, 253-291 (1998)

4. Skenderis, K., van Rees, B.C.: Real-time gauge/gravity duality. Phys. Rev. Lett. 101, 081601 (2008)

5. Skenderis, K., van Rees, B.C.: Real-time gauge/gravity duality: Prescription, Renormalization and Examples. http://arxiv.org/abs/0812.2909v2 [hep-th], 2009

6. Schwinger, J.S.: Brownian motion of a quantum oscillator. J. Math. Phys. 2, 407-432 (1961)

7. Bakshi, P.M., Mahanthappa, K.T.: Expectation value formalism in quantum field theory. 1. J. Math. Phys. 4, 1-11 (1963)

8. Bakshi, P.M., Mahanthappa, K.T.: Expectation value formalism in quantum field theory. 2. J. Math. Phys. 4, 12-16 (1963)

9. Keldysh, L.V.: Diagram technique for nonequilibrium processes. Zh. Eksp. Teor. Fiz. 47, 1515-1527 (1964) [Sov. Phys. JETP 20, 1018 (1965)]

10. Hartle, J.B., Hawking, S.W.: Wave Function of the Universe. Phys. Rev. D 28, 2960-2975 (1983)

11. Maldacena, J.M.: Eternal black holes in Anti-de-Sitter.. JHEP 04, 021 (2003)

12. Aminneborg, S., Bengtsson, I., Brill, D., Holst, S., Peldan, P.: Black holes and wormholes in $2+1$ dimensions. Class. Quant. Grav. 15, 627-644 (1998)

13. Brill, D.: Black holes and wormholes in 2+1 dimensions. http://arxiv.org/abs/gr-qc/9904083v2, 1999

14. Skenderis, K., Solodukhin, S.N.: Quantum effective action from the AdS/CFT correspondence. Phys. Lett. B 472, 316-322 (2000)

15. Krasnov, K.: Holography and Riemann surfaces. Adv. Theor. Math. Phys. 4, 929-979 (2000)

16. Henningson, M., Skenderis, K.: Holography and the Weyl anomaly. Fortsch. Phys. 48, 125-128 (2000)

17. Henningson, M., Skenderis, K.: The holographic Weyl anomaly. JHEP 07, 023 (1998)

18. de Haro, S., Solodukhin, S.N., Skenderis, K.: Holographic reconstruction of spacetime and renormalization in the AdS/CFT correspondence. Commun. Math. Phys. 217, 595-622 (2001)

19. Skenderis, K.: Asymptotically Anti-de Sitter spacetimes and their stress energy tensor. Int. J. Mod. Phys. A 16, 740-749 (2001)

20. Banados, M., Teitelboim, C., Zanelli, J.: The Black hole in three-dimensional space-time. Phys. Rev. Lett. 69, 1849-1851 (1992)

21. Banados, M., Henneaux, M., Teitelboim, C., Zanelli, J.: Geometry of the (2+1) black hole. Phys. Rev. D 48, 1506-1525 (1993)

22. Barbot, T.: Causal properties of AdS-isometry groups. I: Causal actions and limit sets. Adv. Theor. Math. Phys. 12, 1-66 (2008) 
23. Barbot, T.: Causal properties of AdS-isometry groups. II: BTZ multi black-holes. http://arxiv.org/abs/ math.gt/0510065v2 [math, GT], 2006

24. van Rees, B.: Worm holes in 2+1 dimensions. Master's thesis, http://staff.science.uva.nl/ brees/report/ report.pdf June, 2006

25. Imayoshi, Y., Taniguchi, M.: An Introduction to Teichmnüller Spaces. Berlin-Heidelberg Newyork: Springer-Verlag, 1992

26. Lehto, O.: Univalent Functions and Teichmüller Spaces. Berlin-Heidelberg Newyork: Springer-Verlag, 1986

27. Nag, S.: The Complex Analytic Theory of Teichmüller spaces. Newyork: John Wiley \& Sons, 1988

28. Galloway, G.J., Schleich, K., Witt, D.M., Woolgar, E.: Topological Censorship and Higher Genus Black Holes. Phys. Rev. D60, 104039 (1999)

29. Carlip, S., Teitelboim, C.: Aspects of black hole quantum mechanics and thermodynamics in $(2+1)-$ dimensions. Phys. Rev. D51, 622-631 (1995)

30. Krasnov, K.: On holomorphic factorization in asymptotically AdS 3D gravity. Class. Quant. Grav. 20, 4015-4042 (2003)

31. Krasnov, K.: Black Hole Thermodynamics and Riemann Surfaces. Class. Quant. Grav. 20, 2235-2250 (2003)

32. Takhtajan, L., Zograf, P.: On uniformization of Riemann surfaces and the Weyl-Peterson metric on Teichmuller and Schottky spaces. Math. USSR Sbornik 60, 297-313 (1988)

33. Imbimbo, C., Schwimmer, A., Theisen, S., Yankielowicz, S.: Diffeomorphisms and holographic anomalies. Class. Quant. Grav. 17, 1129-1138 (2000)

34. Maldacena, J.M., Maoz, L.: Wormholes in AdS. JHEP 02, 053 (2004)

35. Parlier, H.: Fixed point free involutions on Riemann surfaces. Israel J. Math. 166, 297-311 (2008)

36. Anderson, M.T.: Geometric aspects of the AdS/CFT correspondence. http://arxiv.org/abs/hep-th/ 0403087v2, 2004

37. Papadimitriou, I., Skenderis, K.: Thermodynamics of asymptotically locally AdS spacetimes. JHEP 08, 004 (2005)

38. Freivogel, B., et al.: Inflation in AdS/CFT. JHEP 03, 007 (2006)

39. Louko, J., Marolf, D.: Single-exterior black holes and the AdS-CFT conjecture. Phys. Rev. D59, 066002 (1999)

40. Hawking, S.W., Page, D.N.: Thermodynamics of black holes in anti-de Sitter space. Commun. Math. Phys. 87, 577 (1983)

41. Maldacena, J.M., Strominger, A.: AdS(3) black holes and a stringy exclusion principle. JHEP 12, 005 (1998)

42. Yin, X.: Partition Functions of Three-Dimensional Pure Gravity. http://arxiv.org/abs/0710.2129v2 [hepth] (2008)

43. Aminneborg, S., Bengtsson, I., Holst, S.: A spinning Anti-de Sitter wormhole. Class. Quant. Grav. 16, 363382 (1999)

44. Brill, D.: 2+1-dimensional black holes with momentum and angular momentum. Annalen Phys. 9, 217-226 (2000)

45. Krasnov, K.: Analytic continuation for asymptotically AdS 3D gravity. Class. Quant. Grav. 19, 23992424 (2002)

46. Maloney, A.: To appear

47. Skenderis, K., Taylor, M.: The fuzzball proposal for black holes. Phys. Rept. 467, 117-171 (2008)

48. Crnkovic, C., Witten, E.: Covariant description of canonical formalism in geometrical theories. Print-861309 (Princeton)

49. Crnkovic, C.: Symplectic geometry and (super)Poincare algebra in geometrical theories. Nucl. Phys. B 288, 419 (1987)

50. Lee, J., Wald, R.M.: Local symmetries and constraints. J. Math. Phys. 31, 725-743 (1990)

51. van Rees, B.: Dynamics and the gauge/gravity duality. $\mathrm{PhD}$ thesis, 2010, to appear

Communicated by P.T. Chruściel 